\title{
Hierarchical Task Assignment Strategy for Heterogeneous Multi- UAV System in Large-Scale Search and Rescue Scenarios
}

\author{
Jie Chen $\mathbb{D}$, ${ }^{1}$ Kai Xiao $\mathbb{D}$, ${ }^{1}$ Kai You $\mathbb{D},{ }^{1}$ Xianguo Qing $\mathbb{D},{ }^{1}$ Fang Ye $\mathbb{D}^{2}$, and Qian Sun $\mathbb{D}^{2}$ \\ ${ }^{1}$ Science and Technology on Reactor System Design Technology Laboratory, Nuclear Power Institute of China, \\ Chengdu 610213, China \\ ${ }^{2}$ College of Information and Communication Engineering, Harbin Engineering University, Harbin 150001, China
}

Correspondence should be addressed to Jie Chen; chenjie0229@outlook.com

Received 23 April 2021; Revised 16 June 2021; Accepted 22 June 2021; Published 16 July 2021

Academic Editor: Chen Pengyun

Copyright ( 2021 Jie Chen et al. This is an open access article distributed under the Creative Commons Attribution License, which permits unrestricted use, distribution, and reproduction in any medium, provided the original work is properly cited.

For the large-scale search and rescue (S\&R) scenarios, the centralized and distributed multi-UAV multitask assignment algorithms for multi-UAV systems have the problems of heavy computational load and massive communication burden, which make it hard to guarantee the effectiveness and convergence speed of their task assignment results. To address this issue, this paper proposes a hierarchical task assignment strategy. Firstly, a model decoupling algorithm based on density clustering and negotiation mechanism is raised to decompose the large-scale task assignment problem into several nonintersection and complete smallscale task assignment problems, which effectively reduces the required computational amount and communication cost. Then, a cluster head selection method based on multiattribute decision is put forward to select the cluster head for each UAV team. These cluster heads will communicate with the central control station about the latest assignment information to guarantee the completion of S\&R mission. At last, considering that a few targets cannot be effectively allocated due to UAVs' limited and unbalanced resources, an auction-based task sharing scheme among UAV teams is presented to guarantee the mission coverage of the multi-UAV system. Simulation results and analyses comprehensively verify the feasibility and effectiveness of the proposed hierarchical task assignment strategy in large-scale S\&R scenarios with dispersed clustering targets.

\section{Introduction}

Due to the advantages of no casualties, high flexibility, strong maneuverability, etc. [1-3], unmanned aerial vehicles (UAVs) are widely used in various search and rescue (S\&R) scenarios, e.g., earthquake-hit areas or forest fire zones. Single UAV with limited capability and capacity cannot guarantee the multidimensional and extensive coverage of mission area $[4,5]$. In order to carry out timely search and rescue to survivors in the S\&R scenarios, the cooperative task assignment strategy for multi-UAV system is studied in this paper [6].

For multi-UAV system in the S\&R scenarios, the cooperative multi-UAV task assignment problem (CMTAP) has two characteristics: the time coupling constraints of tasks and the heterogeneity of UAVs.
(1) Each survivor needs to perform search $(S)$ and rescue $(R)$ tasks in sequence to achieve effective rescue. Thus, for the same target, the execution of $S$ and $R$ tasks needs to satisfy the task precedence constraint and task completion constraint. Besides, to ensure timely treatment of each survivor, its $S$ and $R$ tasks need to be carried out within a certain time window

(2) Different UAVs have different capabilities. Search UAVs equipped with various reconnaissance sensors can perform $S$ tasks to assessing damage and monitoring the environment, while rescue UAVs equipped with drugs, food, and other resources can perform $R$ tasks to retrieve the wounded or dying survivors

To solve the CMTAP model in the S\&R scenarios, many scholars have raised two kinds of task assignment methods: 
centralized and distributed algorithms. However, these two kinds of algorithms both have some limitations in the largescale S\&R scenarios.

CMTAP is a nondeterministic polynomial-time hard (NP-hard) combinational optimization problem [7, 8]. Centralized task assignment methods including the optimization methods (decision tree method [9], dynamic programming [10], etc.) and the intelligent optimization algorithms (ant colony algorithm $[11,12]$, particle swarm optimization algorithm [13, 14], genetic algorithm [15-17], etc.) depend on the central control station to make the execution plan for the multi-UAV system. With the increase of CMTAP scale, the optimization methods are faced with the risk of exponentially computational burden $[18,19]$. [20] shows that when 2 UAVs need to perform search and strike mission against 3 targets, the optimization method can quickly search the entire solution space and obtain the optimal solution. However, when 8 UAVs are assigned to perform search and strike mission against 5 targets, the optimization method takes more than three weeks to produce the task assignment solution. Then, CMTAP is a combinational optimization problem with multiple constraints, and the intelligent optimization algorithms rely on iterations to obtain quick task assignment solutions. However, with the increase of CMTAP scale, there will be a large number of invalid solutions that violate the constraints in the iteration process, which seriously affects the efficiency of algorithms [4].

Distributed task assignment algorithms including auction-based algorithm [21, 22], contract net method [23, 24], and consensus-based bundle algorithm (CBBA) [2527] can produce timely execution plan for the multi-UAV system through the continuous communication and negotiation among all UAVs. The auction-based algorithm and contract net method have low communication efficiency and large communication cost, which is not conducive to realizing quick response in large-scale S\&R scenarios [21]. CBBA has been developed into many extensions. [28] presents the grouped CBBA to improve the communication efficiency when ensuring the robustness and convergence of decentralized task assignment solution. Considering the scenario that some tasks must be allocated, [29] raises an extended CBBA to solve the decentralized task assignment problem with critical tasks. Further considering the fuel or time constraints in real-time scenarios, [30] extends CBBA to improve its assignment efficiency for the task allocation problem with spatially and temporally constraints. Besides, [31] evolves the swarmgeneralized assignment problem method for better suitability for solving the task allocation problem in dynamic scenarios.

Overall, the centralized and distributed task assignment algorithms have been greatly developed to solve different task assignment problems for multi-UAV systems. However, with the increase of CMTAP scale, the multi-UAV systems are faced with frequent and massive communication burden $[32,33]$. Neither centralized nor distributed algorithms can produce real-time solution for the large-scale multi-UAV multitask assignment problem.

Besides, in practical large-scale S\&R scenarios, survivors tend to gather at different locations $[34,35]$. That is, there will be dispersed clustering targets in the large-scale S\&R sce- narios. To realize the prompt response in the large-scale S\&R scenarios with dispersed clustering targets, a hierarchical task assignment strategy is proposed to handle the multi-UAV multitask assignment problem.

To effectively solve the task assignment problem in the large-scale S\&R scenarios with dispersed clustering targets, the proposed hierarchical task assignment strategy is applied by the following procedures.

(1) Firstly, a model decoupling algorithm based on density clustering and negotiation mechanism is proposed to effectively decompose the whole task assignment model into several nonintersection and complete submodels with partial targets and UAVs. Then, the algorithm named CBBA with task coupling constraints [36] is applied to solve the local task assignment problem in each submodel. These two procedures ensure that the hierarchical task assignment strategy can produce conflict-free solution satisfying the time coupling constraints of tasks and the heterogeneity of UAVs in S\&R scenarios and simultaneously reduces the computational amount and communication cost

(2) After that, the cluster head selection method based on multiattribute decision is put forward to select the cluster head for each UAV team according to the overall analyses of UAVs' four factors including the mission execution time, the number of its undertaken tasks, the total score value, and the residual task capacity loads. These cluster head will communicate with the central control station about the local task assignment information to ensure the effective operation of S\&R mission

(3) Finally, to realize the mission coverage in the S\&R scenarios, an auction-based task sharing scheme among UAV teams is raised to assign the tasks that cannot be allocated within their submodels. The central control station and the cluster heads will participate in the auction process of unallocated targets to achieve the effective assignment of all tasks in the mission area

The rest of this paper is constructed as follows. Section 2 illustrates the multi-UAV multitask assignment problem in the S\&R scenarios and introduces the corresponding solution algorithm: CBBA with task coupling constraints. Then, Section 3 elaborates the hierarchical task assignment strategy including the hierarchical task assignment model, the model decoupling process, the cluster head selection, and the task sharing scheme. Simulations are given in Section 4 to demonstrate the effectiveness of the proposed hierarchical task assignment strategy in the large-scale S\&R scenarios with dispersed clustering targets. At last, Section 5 concludes this paper.

\section{Multi-UAV Multitask Assignment Problem}

In the S\&R scenarios, heterogeneous multiple UAVs need to successively perform search $(S)$ and rescue $(R)$ tasks on 
multiple stationary ground targets. Notably, in this paper, we assume that the mission area has been scouted, and the information (number, locations, etc.) of survivors is known [37, 38].

2.1. UAVs. Assume that there are $N_{U}$ heterogeneous UAVs in the multi-UAV system, the set of UAVs is

$$
\mathbf{U}=\left\{U_{1}^{\text {type }}, U_{2}^{\text {type }}, \cdots, U_{N_{U}}^{\text {type }}\right\},
$$

where the superscript type $\in\{S, R\}$ respectively denotes the search and rescue UAVs.

The heterogeneity of UAVs reflects on different capabilities and kinematics parameters.

(1) Firstly, search UAV (S-UAV) equipped with various reconnaissance sensors is only capable to perform $S$ task, while rescue UAV ( $R$-UAV) equipped with drugs, food, and other resources is only capable to perform $R$ task

(2) Then, different UAV has different cruise speed and fuel consumption rate

$$
\begin{aligned}
& \mathbf{v}=\frac{\left\{v_{1}, v_{2}, \cdots, v_{N_{U}}\right\} m}{s}, \\
& \mathbf{f}=\frac{\left\{f_{1}, f_{2}, \cdots, f_{N_{U}}\right\}}{k m} .
\end{aligned}
$$

The cruise speeds and fuel consumption rates of UAVs are respectively introduced to describe the flight cost of UAVs and the fuel cost of UAVs. [39] reveals that the fuel consumption rate of actual flight is close to be linear to UAV's flight distance. Thus, the fuel consumptions of UAVs are assumed to be constant in this paper.

Besides, to simulate the practical S\&R scenarios, assume that $\mathrm{UAV} U_{i}\left(i=1,2, \cdots, N_{U}\right)$ can be assigned a maximum of $L_{t}^{i}$ tasks. That is, the task capacity of each UAV is limited.

2.2. Tasks. Suppose that there are $N_{T}$ stationary ground targets in the S\&R mission, each target has two tasks $M_{T}=\{S$, $R\}$ ( $S, R$ separately represent the search and rescue tasks) need to be performed successively. The set of tasks is

$$
T=\left\{T_{1}^{S}, T_{2}^{S}, \cdots, T_{N_{T}}^{S}, T_{1}^{R}, T_{2}^{R}, \cdots, T_{N_{T}}^{R}\right\}=\left\{T_{1}, T_{2}, \cdots, T_{N_{t}}\right\},
$$

where $T_{j}^{S}, T_{j}^{R}\left(j=1,2, \cdots, N_{T}\right)$ separately represent the $S, R$ tasks of target $j$ and $N_{t}=2 N_{T}$ is the number of tasks.

The time coupling constraints of tasks in the S\&R scenarios reflect on task precedence constraint, task completion constraint, and time-sensitive constraint.

(1) For each target, the execution of two tasks should follow strict task precedence constraint that $R$ task can be performed only if $S$ task is completed
(2) The task completion constraint means that $S$ and $R$ tasks should be both assigned to guarantee the successful rescue of certain survivor

(3) To ensure the effective rescue of each survivor, its $S$ and $R$ tasks should be performed within certain time window

Suppose that the performing times of $S$ and $R$ tasks of target $j$ are separately $t_{j}^{S}, t_{j}^{R}$, the time window of target $j$ is [ $\left.t_{j}^{\text {start }}, t_{j}^{\text {end }}\right]$, the execution durations of $S$ and $R$ tasks are separately $t_{\Delta 1}, t_{\Delta 2}$; the time coupling constraints can be defined as follows.

$$
\begin{gathered}
t_{j}^{\text {start }} \leq t_{j}^{S}<t_{j}^{R} \leq t_{j}^{\text {end }}, \\
t_{j}^{S}+t_{\Delta 1} \leq t_{j}^{R}, \\
t_{j}^{R}+t_{\Delta 2} \leq t_{j}^{\text {end }} .
\end{gathered}
$$

2.3. Task Assignment Model. The multi-UAV multitask assignment problem in the S\&R scenarios is described as $N_{U}$ heterogeneous UAVs need to consecutively perform $S$ and $R$ tasks on $N_{T}$ stationary ground targets (total $N_{t}=2$ $N_{T}$ tasks). The objective of the task assignment model is to find a conflict-free matching of tasks to UAVs that maximizes the global reward and simultaneously satisfies the time coupling constraints of tasks and heterogeneity of UAVs.

$$
\max \sum_{i=1}^{N_{U}}\left(\sum_{j=1}^{N_{t}} c_{i j}\left(x_{i}, p_{i}\right) x_{i j}\right),
$$

subject to

$$
\begin{gathered}
\sum_{j=1}^{N_{t}} x_{i j} \leq L_{t}^{i}, \\
\sum_{i=1}^{N_{U}} \sum_{j=1}^{N_{t}} x_{i j} \leq N_{\min } \triangleq\left\{N_{t}, \sum_{i=1}^{N_{U}} L_{t}^{i}\right\} \\
\sum_{i=1}^{N_{U}} x_{i j} \leq 1, \\
\sum_{i=1}^{N_{U}} x_{i\left(j+N_{T}\right)} \leq 1, \\
\sum_{i=1}^{N_{U}} x_{i j}+\sum_{i=1}^{N_{U}} x_{i\left(j+N_{T}\right)}=\{0,2\} \\
t_{j}^{\mathrm{start}} \leq t_{j}<t_{j+N_{T}} \leq t_{j}^{\mathrm{end}}, \\
t_{j}+t_{\Delta 1} \leq t_{j+N_{T}}, \\
t_{j+N_{T}}+t_{\Delta 2} \leq t_{j}^{\mathrm{end}},
\end{gathered}
$$


According to (4), $T_{j}, T_{j+N_{T}} \in \mathbf{T}$ separately represents $S$ and $R$ tasks of target j. $x_{i j}, x_{i\left(j+N_{T}\right)} \in\{0,1\}$ is the decision variable. $x_{i j}, x_{i\left(j+N_{t} / 2\right)}=1$ if and only if task $T_{j}, T_{j+N_{t} / 2}$ is allocated to UAV $U_{i} \in \mathrm{U} . x_{i} \in\{0,1\}^{N_{t}}$ is the decision vector, whose $j^{\text {th }}$ element is $x_{i j} \cdot p_{i} \in(T \cup\{\varnothing\})^{L_{t}^{i}}$ represents the ordered task sequence of UAV $U_{i}$, whose $k^{\text {th }}$ element is $T_{j}$ if UAV $U_{i}$ conducts task $T_{j}$ at $k^{\text {th }}$ waypoint along its task path, and becomes $\varnothing$ (empty task) if UAV conducts less than $k$ tasks. The scoring function $c_{i j}\left(x_{i}, p_{i}\right) \geq 0$ is assumed to be a certain nonnegative function of $x_{i}$ or its path $p_{i}$. The summation term inside the first parenthesis represents the local reward for $\operatorname{UAV} U_{i}$, which is the sum of the rewards of its assigned tasks in path $p_{i}$

The multi-UAV multitask assignment problem described above satisfies the time coupling constraints of tasks and the heterogeneity of UAVs in the S\&R scenarios.

(1) The decision variable $x_{i j}, x_{i\left(j+N_{T}\right)}$ should meet the constraint on different capabilities of UAVs. That is, only $S$-UAV can perform $S$ task of target $j$, and only $R$-UAV can perform its $R$ task

(2) The different kinematics parameters of UAVs reflect on different cruise speed and fuel consumption rate, thus reflect on the scoring function $c_{i j}\left(x_{i}, p_{i}\right)$ in the task assignment model. The scoring value of UAV $U_{i}$ performing task $T_{j}$ is defined as

$$
\left.c_{i j}\left(x_{i}, p_{i}\right) x_{i j}=R_{j 0}+e^{-\lambda_{j}\left(t_{i j}-t_{j}^{\text {start }}\right.}\right) R_{j} u\left(t_{i j}\right)-f_{i} \Delta D_{i j},
$$

where $R_{j 0}, R_{j}\left(j=1,2, \cdots, N_{t}\right)$ separately represents the fixed reward and initial reward associated with task $T_{j}, \lambda_{j}$ is the value decrement factor, $t_{i j}$ is the execution time of task $T_{j}$ in UAV $U_{i}$ 's path $p_{i}, u_{i j}$ is the binary variable that shows if the constraint of time window is satisfied (defined below), and $\Delta D_{i j}$ is the distance from UAV $U_{i}$ 's initial location to task $T_{j}$ 's location.

$$
u\left(\mathrm{t}_{i j}\right)= \begin{cases}1 & \text { if } t_{j}^{\text {start }} \leq t_{i j} \leq t_{j}^{\mathrm{end}} \\ 0 & \text { otherwise }\end{cases}
$$

(3) Considering the limited payload of UAVs, each UAV has limited capacity (9). That is, UAV $U_{i}$ can perform a maximum of $L_{t}^{i}$ limited tasks

(4) We can see from (13) that the $S$ and $R$ tasks of each target should be both assigned; otherwise, the target cannot be effectively rescued. Thus, task completion constraint is well described

(5) The task precedence constraint and time-sensitive constraint are reflected on (14)-(16). That is, $S$ and $R$ tasks of target $j$ should be performed in sequence, and both $S$ and $R$ tasks should be performed in certain time window

2.4. CBBA-TCC. To solve the multi-UAV multitask assignment problem with time coupling constraints of tasks and the heterogeneity of UAVs in the S\&R scenarios, an extension of CBBA, named consensus-based bundle algorithm with task coupling constraints (CBBA-TCC), has been raised in [29]. The flow diagram of CBBA-TCC is given in Figure 1. Details about CBBA-TCC please see [29].

CBBA-TCC can realize conflict-free task assignment through the iterations between inner and outer consensus stages. The inner consensus stage can guarantee that the task assignment solution meets the task precedence constraint and time-sensitive constraint in (14)-(16), while the outer consensus stage is raised to ensure that the task completion constraint in (13) is satisfied. [29] proves that CBBA-TCC can produce feasible and conflict-free task assignment solution in the S\&R scenarios. Therefore, the multi-UAV multitask assignment problem in Section 2.3 is properly solved.

Take the communication topology of the multi-UAV system as an undirected graph, the shortest path between two UAVs $U_{i}, U_{k}\left(i, k=1,2, \cdots, N_{U}\right)$ is $d_{i k}<\propto$. Thus, the network diameter of the communication topology is defined as

$$
D=\max _{i, k=1,2, \cdots, N_{U}} d_{i k}
$$

Accordingly, each UAV needs to communicate a maximum of $D$ times to get consensus solution. Besides, the worse situation is that only one task is assigned in one iteration of communication and negotiation. Thus, multi-UAV system needs a maximum of $N_{\text {min }}$ iterations to get global task assignment result. Therefore, the maximum communication amount of CBBA-TCC to produce feasible and conflict-free task assignment solution is

$$
Y=N_{\min } D
$$

\section{Hierarchical Task Assignment Strategy}

In the large-scale S\&R scenarios, survivors tend to gather in different locations. Due to potential problems of exponentially computational complexity and massive communication burden, it is impractical to use centralized or distributed task assignment algorithms to produce real-time task assignment solutions for the large-scale S\&R scenarios. Thus, the hierarchical task assignment strategy is proposed for the large-scale S\&R scenarios with dispersed clustering targets.

Firstly, the hierarchical task assignment model of the large-scale S\&R scenarios is discussed. The theoretical analysis about the communication amount of the hierarchical task assignment strategy is deduced to prove the effectiveness of the proposed algorithm in the large-scale $S \& R$ scenarios. Then, the proposed hierarchical task assignment strategy consists of three main innovations: model decoupling of the large-scale task assignment problem, cluster head selection of $N_{C}$ submodels, and the task sharing scheme among 


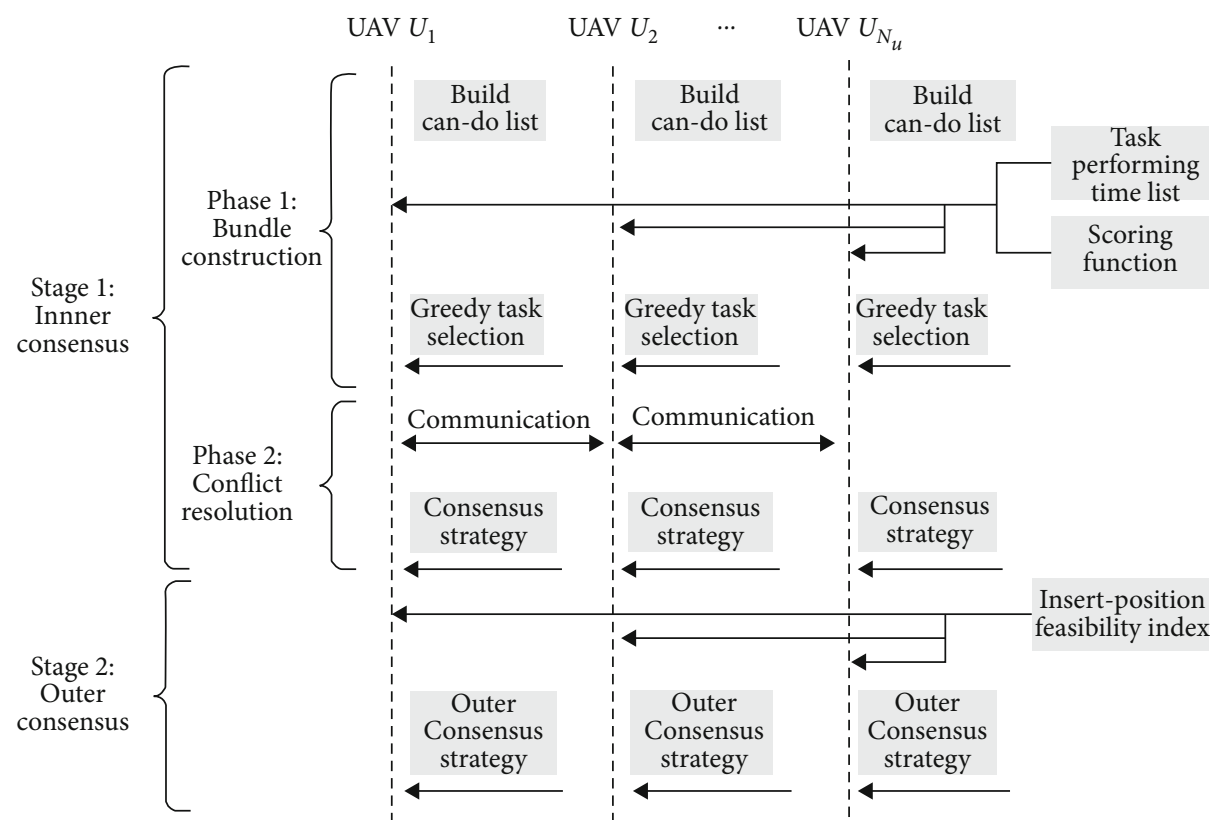

Figure 1: CBBA-TCC.

multiple UAV teams. Thus, these three procedures of the proposed algorithm are also elaborated.

3.1. Hierarchical Task Assignment Model. Firstly, the largescale task assignment model with $N_{U}$ UAVs and $N_{T}$ targets can be decomposed into $N_{C}$ submodels. The UAV set and target set in these submodels are defined as $U_{1}, U_{2}, \cdots, U_{N_{C}}$ and $T_{1}, T_{2}, \cdots, T_{N_{C}}$.

To produce conflict-free solution for the whole task assignment problem, UAVs and targets in these submodels should be nonintersection and complete.

$$
\begin{gathered}
U_{p} \cap U_{q}=\varnothing, \\
U_{1} \cup U_{2} \cup \cdots \cup U_{N_{C}}=U, \\
\left\|U_{1}\right\|+\left\|U_{2}\right\|+\cdots+\left\|U_{N_{C}}\right\|=\|U\|=N_{U}, \\
T_{p} \cap T_{q}=\varnothing, \\
T_{1} \cup T_{2} \cup \cdots \cup T_{N_{C}}=T, \\
\left\|T_{1}\right\|+\left\|T_{2}\right\|+\cdots+\left\|T_{N_{C}}\right\|=\|T\|=N_{T}, \\
\forall p, q=1,2, \cdots, N_{C} .
\end{gathered}
$$

(22) and (23) and (25) and (26) reflect that the total number of UAVs (targets) in all submodels should equal to that of the whole task assignment model. (21) and (24) reflect that there is no overlap of UAVs (targets) among different subteams.

Accordingly, the large-scale task assignment problem with dispersed clustering targets has been decomposed into $N_{C}$ nonintersection and complete submodels.

Then, suppose that the UAV set and task set in $q^{\text {th }}(q=$ $\left.1,2, \cdots, N_{C}\right)$ submodel are separately $U_{q}=\left\{U_{1}^{\text {type }}, U_{2}^{\text {type }}, \cdots\right.$,
$\left.U_{N_{U_{q}}}^{\mathrm{type}}\right\} \quad$ and $\quad T_{q}=\left\{T_{1}^{S}, T_{2}^{S}, \cdots, T_{N_{T_{Q}}}^{S}, T_{1}^{R}, T_{2}^{R}, \cdots, T_{N_{T_{Q}}}^{R}\right\}=\left\{T_{1}\right.$, $\left.T_{2}, \cdots, T_{N_{T_{q}}}\right\}$, the corresponding multi-UAV multitask assignment model is shown below.

$$
\max \sum_{U_{i} \in U_{q}}\left(\sum_{T_{j} \in T_{q}} c_{i j}\left(x_{i}, p_{i}\right) x_{i j}\right),
$$

subject to

$$
\begin{gathered}
\sum_{T_{j} \in T_{q}} x_{i j} \leq L_{t}^{i}, \\
\sum_{U_{i} \in U_{q} T_{j} \in T_{q}} x_{i j} \leq N_{q, \min }=\left\{N_{\left.T_{q}, \sum_{U_{i} \in U_{q}} L_{t}^{i}\right\},}\right. \\
\sum_{U_{i} \in U_{q}} x_{i j} \leq 1, \\
\sum_{U_{i} \in U_{q}} x_{i\left(j+N_{T_{Q}}\right)} \leq 1, \\
\sum_{U_{i} \in U_{q}} x_{i j}+\sum_{U_{i} \in U_{q}} x_{i\left(j+N_{T_{Q}}\right)}=\{0,2\}, \\
t_{j}^{\mathrm{start}} \leq t_{j}<t_{j+N_{T_{Q}}} \leq t_{j}^{\text {end }}, \\
t_{j}+t_{\Delta 1} \leq t_{j+N_{T_{Q}}}, \\
t_{j+N_{T_{Q}}}+t_{\Delta 2} \leq t^{\text {end }},
\end{gathered}
$$

$\forall i=1,2, \cdots, N_{U_{q}}, j=1,2, \cdots, N_{T_{Q}}$, where $N_{U_{q}}, N_{T_{Q}}$, and 
$N_{T_{q}}$ respectively denote the numbers of UAVs, targets, and tasks in $q^{\text {th }}$ submodel.

Apparently, the multi-UAV multitask assignment model in submodel can be solved by CBBA-TCC.

According to (20), the maximum communication amount of $q^{\text {th }}$ submodel is

$$
Y_{q}=N_{q, \min } D_{q}
$$

Then, the maximum communication amount of the hierarchical task assignment model is

$$
\begin{aligned}
Y^{\prime} & =\sum_{q=1}^{N_{C}} Y_{q}=\sum_{q=1}^{N_{C}}\left(N_{q, \min } D_{q}\right) \leq \sum_{q=1}^{N_{C}}\left(N_{q, \min } D\right)=\left(\sum_{q=1}^{N_{C}} N_{q, \min }\right) \times D \\
& =N_{\min } D=Y .
\end{aligned}
$$

Obviously, through effective model decomposition, the hierarchical task assignment model has less communication requirements than the original task assignment model. Notably, when $N_{C}$ is large, there will be a great deal of submodels. Under this circumstance, $Y^{\prime} \ll Y$; thus, the required communication amount is well reduced.

\subsection{Model Decoupling Algorithm Based on Density Clustering} and Negotiation Mechanism. Last section proves that the hierarchical task assignment model effectively reduces the required communication amount. Thus, the core is to study the dimensional reduction method that can break the largescale task assignment model with dispersed clustering targets into several nonintersection and complete submodels. To address this issue, a model decoupling algorithm based on density clustering and negotiation mechanism (MDADCNM) is put forward. The schematic diagram of MDADCNM is given in Figure 2.

According to Figure 2, MDA-DCNM consists of two procedures. Firstly, the density clustering method is applied to effectively cluster these dispersed clustering targets. Then, according to UAVs' limited resources and different task requirements of these clustered targets, negotiation mechanism is utilized to form heterogeneous UAV teams for these clustered targets. Therefore, the large-scale task assignment problem with dispersed clustering targets can be decomposed into several nonintersection and complete small-scale task assignment problems, which effectively reduces the required computational amount and communication cost.

3.2.1. Density Clustering of Targets. According to the distribution of targets in the mission area, the central control station firstly introduces the density clustering method to divide $N_{T}$ targets into $N_{C}$ subclusters. Since density clustering method finds different clusters by constantly connecting high-density points in the neighborhood, there are only two parameters needed: neighborhood size MinPts and density threshold $\varepsilon$. Thus, different from $K$-mean clustering method, density clustering method can find clusters with different shapes and scales, which is suitable to divide these dispersed clustering targets into multiple different subteams [40, 41].
The algorithmic flow of density clustering method in this paper is shown in Algorithm 1.

Therefore, $N_{T}$ targets have been clustered into $N_{C}$ target sets: $T_{1}, T_{2}, \cdots, T_{N_{C}}$. The task sets in these target clusters are $\bar{T}_{1}, \bar{T}_{2}, \cdots, \bar{T}_{N_{C}}$, where $q=1,2, \cdots N_{C}, \bar{T}_{q}=\left\{T_{1}^{S}, T_{2}^{S}, \cdots, T_{N_{T_{Q}}}^{S}\right.$, $\left.T_{1}^{R}, T_{2}^{R}, \cdots, T_{N_{T_{Q}}}^{R}\right\}$.

3.2.2. Negotiation-Based Assignment of UAVs. Different UAVs have different limited task capacities, and different subclusters have different number of targets. According to the different requirements of tasks in different subclusters, negotiation-based assignment algorithm is proposed to allocate different UAV teams to these subclusters.

As $N_{T}$ targets have been clustered to $N_{C}$ target sets: $T_{1}$, $T_{2}, \cdots, T_{N_{C}}$, the requirements for $S$ and $R$ tasks in these subclusters are separately defined as

$$
\begin{gathered}
\mathbf{R e}_{S}=\left\{\operatorname{Re}_{1}^{S}, \operatorname{Re}_{2}^{S}, \cdots, \operatorname{Re}_{N_{C}}^{S}\right\}, \\
\mathbf{R e}_{R}=\left\{\operatorname{Re}_{1}^{R}, \operatorname{Re}_{2}^{R}, \cdots, \operatorname{Re}_{N_{C}}^{R}\right\} .
\end{gathered}
$$

Suppose that there are $N_{S} S$-UAVs and $N_{R} R$-UAVs in the multi-UAV system, the UAV sets that can perform $S$ and $R$ tasks are separately defined as

$$
\begin{array}{r}
\mathbf{U}_{S}=\left\{U_{1}, U_{2}, \cdots, U_{N_{S}}\right\}, \\
\mathbf{U}_{R}=\left\{U_{1}, U_{2}, \cdots, U_{N_{R}}\right\} .
\end{array}
$$

In this paper, assume that $S$-UAV has unlimited capacity, while $R$-UAV has limited task capacity due to its limited goods payload. Thus, the negotiation mechanism is only applied to assign $R$-UAVs to these subclusters. After that, $S$ -UAVs will be correspondingly allocated based on the assignment of $R$-UAVs.

Firstly, the limited task capacities of $R$-UAVs are respectively defined as

$$
\mathbf{L}_{t}^{R}=\left\{L_{t}^{1}, L_{t}^{2}, \cdots, L_{t}^{N_{R}}\right\}
$$

The negotiation-based assignment of $R$-UAVs is given in Algorithm 2.

Then, the S-UAV teams of $N_{C}$ submodels can be proportionally assigned based the assignment results of R-UAVs. The number of S-UAVs in $q^{\text {th }}\left(q=1,2, \cdots, N_{C}\right)$ submodel is

$$
N_{U_{q}}^{S}=\frac{N_{U_{q}}^{R}}{\sum_{p=1}^{N_{C}} N_{U_{p}}^{R}} \times N_{S} .
$$

An example is given to illustrate the negotiation-based assignment of UAVs.

Suppose that the whole task assignment model with $N_{U}$ $=14 \mathrm{UAVs}$ and $N_{T}=40$ dispersed clustering targets can be divided into $N_{C}=3$ subclusters according to density clustering method, the number of targets in $N_{C}=3$ subteams is $\|$ 


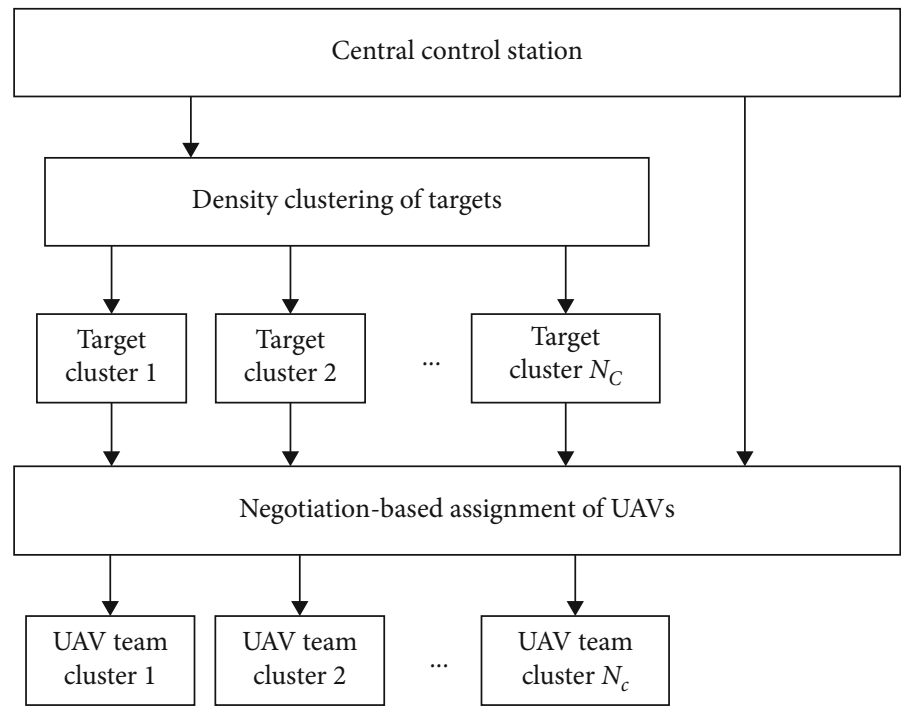

Figure 2: MDA-DCNM.

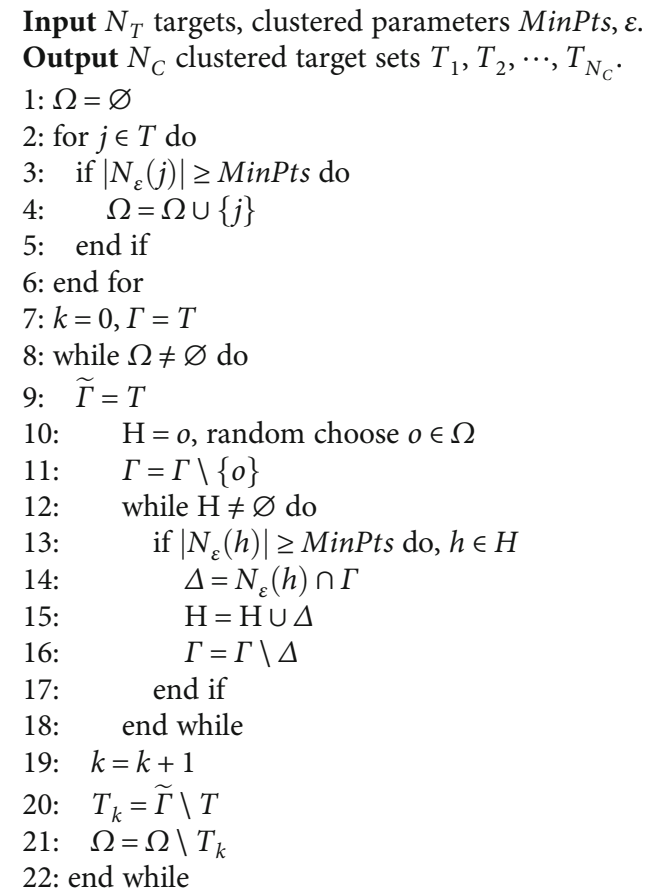

Algorithm 1: Density clustering of targets.

$T_{1}\|=8,\| T_{2}\|=12,\| T_{N_{C}} \|=20$. Thus, the requirements of $R$ tasks in $N_{C}=3$ subclusters are

$$
\mathbf{R e}_{R}=\left\{R e_{1}^{R}, \operatorname{Re}_{2}^{R}, \operatorname{Re}_{3}^{R}\right\}=\{8,12,20\}
$$

Assume that the multi-UAV system has $N_{S}=7 S-U A V s$ and $N_{R}=7 R$-UAVs, the UAV set is

$$
\mathbf{U}=\left\{U_{S}^{1}, U_{S}^{2}, \cdots, U_{S}^{7}, U_{R}^{1}, U_{R}^{2}, \cdots, U_{R}^{7}\right\}
$$

Besides, the limited task capacities of $N_{R}$ R-UAVs are

$$
\mathbf{L}_{t}^{R}=\left\{L_{t}^{1}, L_{t}^{2}, \cdots, L_{t}^{7}\right\}=\{9,10,6,5,7,8,4\} .
$$

Table 1 shows the demonstration of the negotiationbased assignment of $R-\mathrm{UAVs}$.

According to Table 1, the assignment results of $N_{C}=3 R$ -UAV teams are

$$
\begin{array}{r}
\mathbf{U}_{1}^{R}=\left\{U_{5}^{R}, U_{7}^{R}\right\}, \\
\mathbf{U}_{2}^{R}=\left\{U_{1}^{R}, U_{3}^{R}\right\}, \\
\mathbf{U}_{3}^{R}=\left\{U_{2}^{R}, U_{6}^{R}, U_{4}^{R}\right\},
\end{array}
$$

After that, the $N_{C}$ UAV teams can be derived as

$$
\begin{gathered}
\mathbf{U}_{1}=\left\{U_{1}^{S}, U_{2}^{S}, U_{5}^{R}, U_{7}^{R}\right\}, \\
\mathbf{U}_{2}=\left\{U_{3}^{S}, U_{4}^{S}, U_{1}^{R}, U_{3}^{R}\right\}, \\
\mathbf{U}_{3}=\left\{U_{5}^{S}, U_{6}^{S}, U_{7}^{S}, U_{2}^{R}, U_{6}^{R}, U_{4}^{R}\right\} .
\end{gathered}
$$

In Table 1 , after $7^{\text {th }}$ negotiation, $\mathbf{R e}_{R}=\{-3,-3,-3\}$, which means that the residual task capacities of $N_{C}$ UAV teams against targets' requirements on $R$ tasks all equal to 3 . Accordingly, the negotiation-based assignment algorithm can form corresponding UAV teams when balancing the residual task capacities of different UAV teams.

Besides, there is no overlap of UAVs (targets) among different subteams. Therefore, the proposed MDR-DCNM effectively divides the large-scale task assignment model with dispersed clustering targets into multiple small-scale and nonoverlapping task assignment problem.

After that, CBBA-TCC is adopted to solve these smallscale task assignment problems. It is obvious that the solutions of these nonintersection and complete small-scale task assignment problems are conflict-free. Thus, MDR-DCNM 


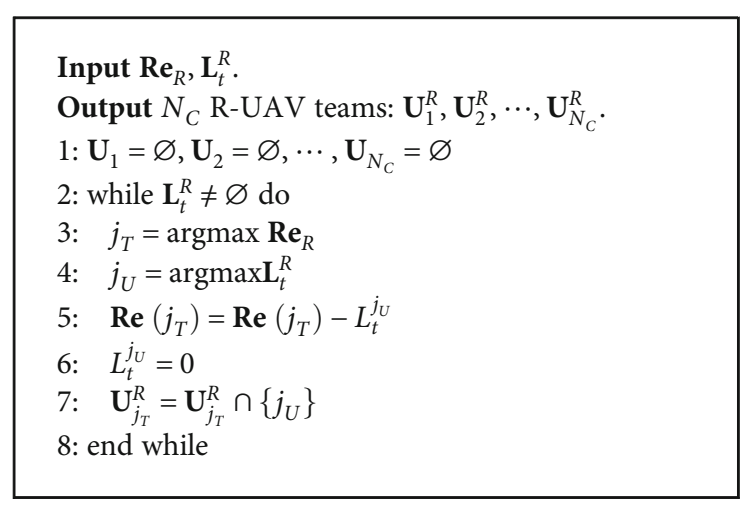

Algorithm 2: Negotiation-based assignment of R-UAVs.

TABLE 1: Demonstration of $R$-UAV allocation based on negotiation mechanism.

\begin{tabular}{|c|c|}
\hline Procedures & Assignment information \\
\hline \multirow{2}{*}{ Step 0} & $\mathbf{R e}_{R}=\{8,12,20\}, \mathbf{L}_{t}^{R}=\{9,10,6,5,7,8,4\}$ \\
\hline & $\mathbf{U}_{1}^{R}=\varnothing, \mathbf{U}_{2}^{R}=\varnothing, \mathbf{U}_{3}^{R}=\varnothing$ \\
\hline \multirow{3}{*}{ Step 1} & $j_{T}=3, j_{U}=2$ \\
\hline & $\mathbf{R e}_{R}=\{8,12,10\}, \mathbf{L}_{t}^{R}=\{9,0,6,5,7,8,4\}$ \\
\hline & $\mathbf{U}_{1}^{R}=\varnothing, \mathbf{U}_{2}^{R}=\varnothing, \mathbf{U}_{3}^{R}=\left\{U_{2}^{R}\right\}$ \\
\hline \multirow{3}{*}{ Step 2} & $j_{T}=2, j_{U}=1$ \\
\hline & $\mathbf{R e}_{R}=\{8,3,10\}, \mathbf{L}_{t}^{R}=\{0,0,6,5,7,8,4\}$ \\
\hline & $\mathbf{U}_{1}^{R}=\varnothing, \mathbf{U}_{2}^{R}=\left\{U_{1}^{R}\right\}, \mathbf{U}_{3}^{R}=\left\{U_{2}^{R}\right\}$ \\
\hline \multirow{3}{*}{ Step 3} & $j_{T}=3, j_{U}=6$ \\
\hline & $\mathbf{R e}_{R}=\{8,3,2\}, \mathbf{L}_{t}^{R}=\{0,0,6,5,7,0,4\}$ \\
\hline & $\mathbf{U}_{1}^{R}=\varnothing, \mathbf{U}_{2}^{R}=\left\{U_{1}^{R}\right\}, \mathbf{U}_{3}^{R}=\left\{U_{2}^{R}, U_{6}^{R}\right\}$ \\
\hline \multirow{3}{*}{ Step 4} & $j_{T}=1, j_{U}=5$ \\
\hline & $\mathbf{R e}_{R}=\{1,3,2\}, \mathbf{L}_{t}^{R}=\{0,0,6,5,0,0,4\}$ \\
\hline & $\mathbf{U}_{1}^{R}=\left\{U_{5}^{R}\right\}, \mathbf{U}_{2}^{R}=\left\{U_{1}^{R}\right\}, \mathbf{U}_{3}^{R}=\left\{U_{2}^{R}, U_{6}^{R}\right\}$ \\
\hline \multirow{4}{*}{ Step 5} & $j_{T}=2, j_{U}=3$ \\
\hline & $\mathbf{R e}_{R}=\{1,-3,2\}, \mathbf{L}_{t}^{R}=\{0,0,0,5,0,0,4\}$ \\
\hline & $\mathbf{U}_{1}^{R}=\left\{U_{5}^{R}\right\}, \mathbf{U}_{2}^{R}=\left\{U_{1}^{R}, U_{3}^{R}\right\}$ \\
\hline & $\mathbf{U}_{3}^{R}=\left\{U_{2}^{R}, U_{6}^{R}\right\}$ \\
\hline \multirow{4}{*}{ Step 6} & $j_{T}=3, j_{U}=4$ \\
\hline & $\mathbf{R e}_{R}=\{1,-3,-3\}, \mathbf{L}_{t}^{R}=\{0,0,0,0,0,0,4\}$ \\
\hline & $\mathbf{U}_{1}^{R}=\left\{U_{5}^{R}\right\}, \mathbf{U}_{2}^{R}=\left\{U_{1}^{R}, U_{3}^{R}\right\}$ \\
\hline & $\mathbf{U}_{3}^{R}=\left\{U_{2}^{R}, U_{6}^{R}, U_{4}^{R}\right\}$ \\
\hline \multirow{4}{*}{ Step 7} & $j_{T}=1, j_{U}=7$ \\
\hline & $\mathbf{R e}_{R}=\{-3,-3,-3\}, \mathbf{L}_{t}^{R}=\{0,0,0,0,0,0,0\}$ \\
\hline & $\mathbf{U}_{1}^{R}=\left\{U_{5}^{R}, U_{7}^{R}\right\}, \mathbf{U}_{2}^{R}=\left\{U_{1}^{R}, U_{3}^{R}\right\}$ \\
\hline & $\mathbf{U}_{3}^{R}=\left\{U_{2}^{R}, U_{6}^{R}, U_{4}^{R}\right\}$ \\
\hline
\end{tabular}

can realize conflict-free task assignment for the large-scale S\&R scenarios.

3.3. Cluster Head Selection Based on Multiattribute Decision. The local task assignment problem in these subclusters has been well solved. Then, the cluster head of each UAV team should send these local task assignment solutions back to central control station for further management. Apparently, the cluster head of each UAV team undertakes extracommunication burden. Besides, since CBBA-TCC depends on communications to generate consensus task assignment solution, the selection of cluster head in each UAV team affects the efficiency of local task assignment. To address this issue, a cluster head selection approach based on multiattribute decision (CHS-MAD) is put forward to choose the cluster head of each UAV team according to the overall analyses of UAVs' four factors including the mission execution time, the number of undertaken tasks, the total score values, and the residual task capacities.

Firstly, suppose that there are $N_{U_{q}}$ UAVs assigned to $q^{\text {th }}\left(q=1,2, \cdots, N_{C}\right)$ submodel, where the number of $S$-UAVs and $R$-UAVs is respectively $N_{N_{U_{q}}}^{S}$ and $N_{N_{U_{q}}}^{R}$, the UAV set can be defined as

$\mathbf{U}_{q}=\left\{U_{1}^{S}, U_{2}^{S}, \cdots, U_{N_{N_{U_{q}}}^{S}}^{S}, U_{1}^{R}, U_{2}^{R}, \cdots, U_{N_{N_{U_{q}}}^{R}}^{R}\right\}=\left\{U_{1}, U_{2}, \cdots, U_{N_{U_{q}}}\right\}$.

According to the local task assignment solution in $q^{\text {th }}$ submodel, four attributes of $N_{U_{q}}$ UAVs can be defined as

$$
\begin{gathered}
t_{U_{q}}=\left\{t_{1}, t_{2}, \cdots, t_{N_{U_{q}}}\right\}, \\
n_{U_{q}}=\left\{n_{1}, n_{2}, \cdots, n_{N_{U_{q}}}\right\}, \\
c_{U_{q}}=\left\{c_{1}, c_{2}, \cdots, c_{N_{U_{q}}}\right\}, \\
\gamma_{U_{q}}=\left\{\gamma_{1}, \gamma_{2}, \cdots, \gamma_{N_{U_{q}}}\right\} .
\end{gathered}
$$

(54) denotes the mission execution time of $N_{U_{q}}$ UAVs. If certain UAV has longer mission execution time, it means that the UAV has less unoccupied time; thus, its probability to be chosen as the cluster head is less.

(55) denotes the number of UAVs' undertaken tasks. If certain UAV has larger number of undertaken tasks, it reflects that the UAV carries out more tasks; thus, its probability to be chosen as the cluster head is less.

(56) denotes the total score value of UAVs. If certain UAV has bigger score value, it means that the UAV has greater roles in the sub-model; thus, its probability to be chosen as the cluster head is less.

(57) denotes the residual task capacities of UAVs. If certain UAV has larger residual task capacity, it reflects that the UAV has stronger flexibility during the task execution; thus, its probability to be chosen as the cluster head is bigger. 
Then, DS evidence theory is adopted to form the multiattribute decision approach. DS evidence theory is a multisource fusion method with quick response and well measurement about uncertain information [42]. Multiattribute decision based on DS evidence theory can comprehensively consider the complementarity and redundancy of four attributes to select the suitable cluster head for each subteam [43]. The DS fusion equation is

$$
M\left(U_{i}\right)=\frac{1}{1-K} \sum_{\cap H=U_{i}}\left(\prod_{1 \leq f \leq 4} m_{f}(H)\right),
$$

where $K$ is the conflict factor that defined as

$$
K=\sum_{n H=\varnothing}\left(\prod_{1 \leq f \leq 4} m_{f}(H)\right) .
$$

The mass functions $m_{f}(H), f=1,2,3,4$ respectively denote UAVs' probabilities to be selected as the clusterhead according to four attributes in (54)-(57).

$$
\begin{aligned}
& m_{1}\left(U_{i}\right)=\frac{t \wedge_{i}^{-2}}{\sum_{p=1}^{N_{U_{q}}} t \wedge_{i}^{-2}}, \\
& m_{2}\left(U_{i}\right)=\frac{n \wedge_{i}^{-2}}{\sum_{p=1}^{N_{U_{q}}} n \wedge_{i}^{-2}}, \\
& m_{3}\left(U_{i}\right)=\frac{c \wedge_{i}^{-2}}{\sum_{p=1}^{N_{U_{q}}} c \wedge_{i}^{-2}}, \\
& m_{4}\left(U_{i}\right)=\frac{\gamma_{i}}{\sum_{p=1}^{N_{U_{q}}} \gamma_{p}},
\end{aligned}
$$

where

$$
\begin{gathered}
\widehat{t}_{i}=\frac{t_{i}}{\max _{i=1,2, \cdots, N_{U_{q}}} t_{i}}, \\
\widehat{n}_{i}=\frac{n_{i}}{\max _{i=1,2, \cdots, N_{U_{q}}} n_{i}}, \\
\widehat{c}_{i}=\frac{c_{i}}{\max _{i=1,2, \cdots, N_{U_{q}}} c_{i}} .
\end{gathered}
$$

Finally, the UAV with largest probability in the fusion result $M\left(U_{i}\right)$ will be chosen as the cluster head of $q^{\text {th }}$ submodel.

$$
J_{i}=\arg \max _{f} M\left(U_{f}\right)
$$

3.4. Auction-Based Task Sharing Scheme among UAV Teams. A problem occurs when a subteam is unable to perform all of its assigned tasks due to UAVs' limited

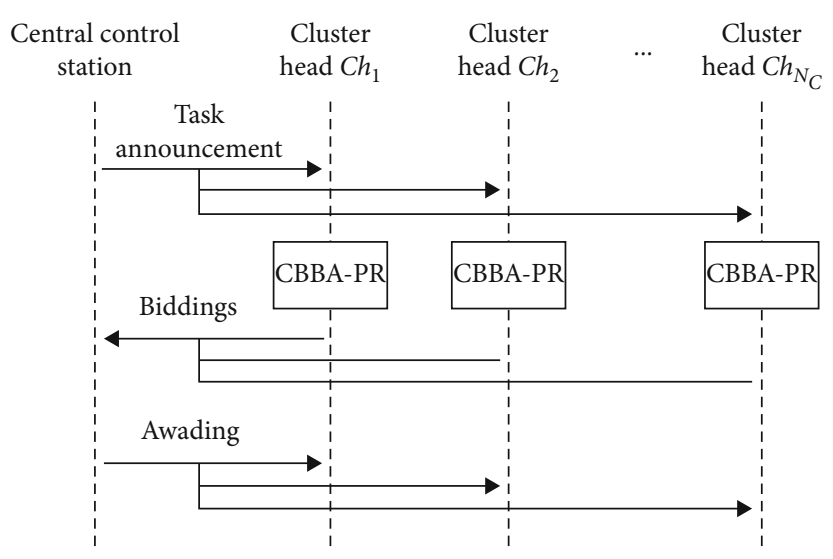

Figure 3: ATS-UT.

TABLE 2: Simulation parameters of UAVs.

\begin{tabular}{lccc}
\hline UAVs & $\begin{array}{c}\text { Task } \\
\text { capacity } L_{t}^{i}\end{array}$ & $\begin{array}{c}\text { Cruise speed } v_{i} \\
(\mathrm{~m} / \mathrm{s})\end{array}$ & $\begin{array}{c}\text { Fuel consumption rate } \\
f_{i}(/ \mathrm{km})\end{array}$ \\
\hline $\begin{array}{l}\text { Search } \\
\text { UAV }\end{array}$ & Unlimited & 80 & 8 \\
$\begin{array}{l}\text { Rescue } \\
\text { UAV }\end{array}$ & Random & 60 & 5 \\
\hline
\end{tabular}

TABLE 3: Simulation parameters of tasks.

\begin{tabular}{lcccc}
\hline Tasks & $\begin{array}{c}\text { Fixed } \\
\text { reward } R_{j 0}\end{array}$ & $\begin{array}{c}\text { Initial } \\
\text { reward } R_{j}\end{array}$ & $\begin{array}{c}\text { Value decrement } \\
\text { factor } \lambda_{j}\end{array}$ & $\begin{array}{c}\text { Execution } \\
\text { duration(s) }\end{array}$ \\
\hline$S$ & 10 & 90 & 0.02 & 5 \\
$R$ & 10 & 90 & 0.02 & 15 \\
\hline
\end{tabular}

and unbalanced resources [44]. Thus, an auction-based task-sharing scheme among UAV teams (ATS-UT) is raised to allocate the unassigned tasks. In the proposed algorithm, the central control station and cluster heads of all UAV teams will participate in the auction process of the unallocated tasks to achieve the effective assignment of all tasks in the mission area and further guarantee the mission coverage of the multi-UAV system in the entire S\&R scenarios. The algorithmic diagram of ATS-UT is shown in Figure 3.

In Figure 3, CBBA-PR represents CBBA with partial replanning that raised in $[45,46]$. CBBA-PR partially resets original task schedules to efficiently and quickly allocate the new task. Thus, CBBA-PR is adopted to solve the allocation of unassigned task in this paper.

Firstly, the central control station will announce the unallocated tasks to all UAV teams once at a time. Then, cluster head of each UAV team will use CBBA-PR to calculate the score increment of the announced task and send it back to the central control station. Finally, the central control station will choose the UAV team with highest score increment to perform the announced task. After iterations of ATS-UT, all unallocated tasks can be well assigned. 


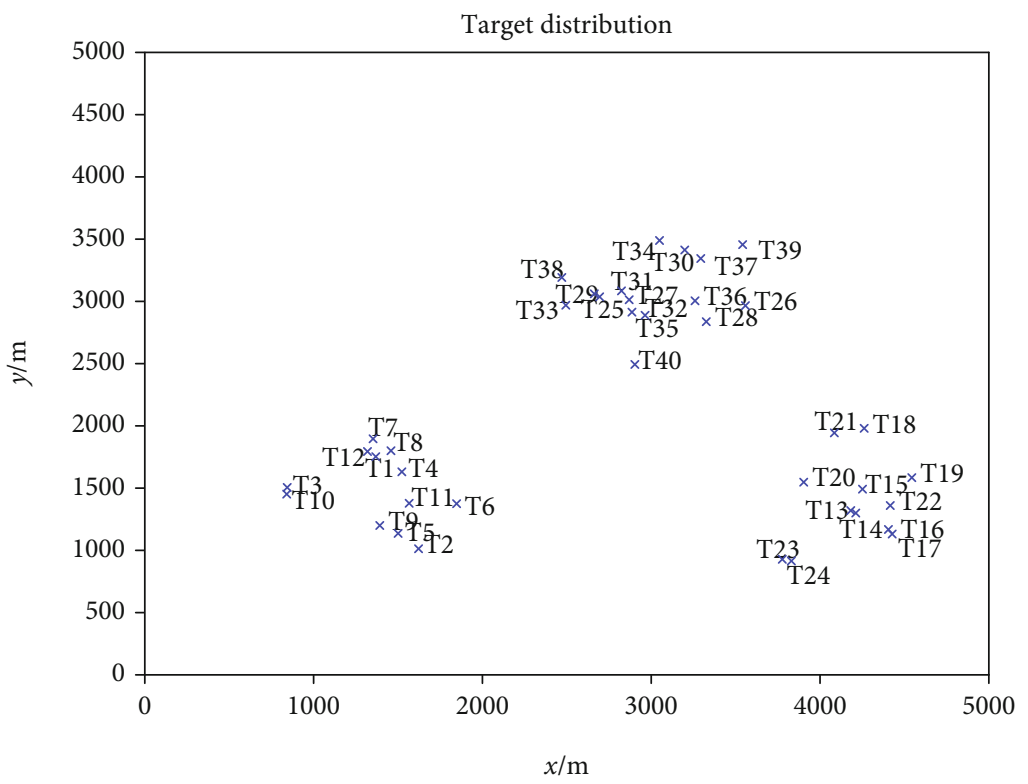

FIgURE 4: $N_{T}=40$ randomly distributed targets.

TABle 4: Relevant elements of $N_{C}=3$ subproblems.

\begin{tabular}{lccc}
\hline Elements & $1^{\text {st }}$ subproblem & $2^{\text {nd }}$ subproblem & $3^{\text {rd }}$ subproblem \\
\hline $\mathbf{T}_{q}$ & $\left\{T_{1}, T_{2}, \cdots, T_{12}\right\}$ & $\left\{T_{13}, T_{14}, \cdots, T_{24}\right\}$ & $\left\{T_{25}, T_{26}, \cdots, T_{40}\right\}$ \\
$R e_{q}^{R}$ & 12 & 12 & 16 \\
$\mathbf{U}_{q}$ & $\left\{U_{1}^{S}, U_{2}^{S}, U_{4}^{R}, U_{7}^{R}\right\}$ & $\left\{U_{3}^{S}, U_{4}^{S}, U_{3}^{R}, U_{1}^{R}\right\}$ & $\left\{U_{5}^{S}, U_{6}^{S}, U_{7}^{S}, U_{5}^{R}, U_{2}^{R}, U_{6}^{R}\right\}$ \\
$\mathbf{L}_{t}^{R}$ & $\{10,7\}$ & $\{9,8\}$ & $\{10,9,7\}$ \\
$\gamma_{q}$ & 5 & 5 & 10 \\
$C h_{q}$ & $U_{2}^{S}$ & $U_{3}^{S}$ & $U_{7}^{S}$ \\
\hline
\end{tabular}

\section{Simulations}

In this paper, MDA-DCNM is the main component of the hierarchical task assignment strategy, which is used to realize model decomposition. CHS-MAD is raised to achieve effective communications between the central control station and multiple UAV teams. ATS-UT in the hierarchical task assignment strategy is only applied when some tasks are not effectively assigned through MDA-DCNM. To prove the feasibility and superiority of the proposed hierarchical task assignment strategy, five simulations are conducted in various large-scale S\&R scenarios.

4.1. Feasibility of $M D A-D C N M$. Suppose that $N_{T}=40$ targets randomly gather at different locations in $5 \mathrm{~km} \times 5 \mathrm{~km}$ mission area, $N_{U}=14 \mathrm{UAVs}$ need to perform S\&R mission on all targets within time window $[0,200] \mathrm{s}$. The UAV set is

$$
\mathbf{U}=\left\{U_{1}^{S}, U_{2}^{S}, \cdots, U_{7}^{S}, U_{1}^{R}, U_{2}^{R}, \cdots, U_{7}^{R}\right\}
$$

where $N_{S}=7, N_{R}=7$.

The simulation parameters of UAVs and tasks are separately shown in Tables 2 and 3.
The randomly distributed targets are shown in Figure 4. The realization of MDA-DCNM is illustrated as follows.

Firstly, the central control station uses density clustering to cluster the dispersed clustering targets in S\&R mission area. Thus, $N_{C}=3$ subclusters are generated. The target sets of $N_{C}=3$ subclusters are

$$
\begin{gathered}
T_{1}=\left\{T_{1}, T_{2}, \cdots, T_{12}\right\}, \\
T_{2}=\left\{T_{13}, T_{14}, \cdots, T_{24}\right\}, \\
T_{3}=\left\{T_{25}, T_{26}, \cdots, T_{40}\right\} .
\end{gathered}
$$

Comparing the clustering results in (69)-(71) with target distribution in Figure 4, we can derive that the dispersed clustering targets have been properly clustered. Besides, we can see from (69) to (71) that:

(1) The numbers of targets in $N_{C}=3$ subclusters are separately $\left\|\mathbf{T}_{1}\right\|=12,\left\|\mathbf{T}_{2}\right\|=12$, and $\left\|\mathbf{T}_{3}\right\|=16$. Apparently, $\quad\left\|\mathbf{T}_{1}\right\|+\left\|\mathbf{T}_{2}\right\|+\left\|\mathbf{T}_{3}\right\|=N_{U}, \quad$ and $\mathbf{T}_{1} \cup \mathbf{T}_{2} \cup \mathbf{T}_{3}=\mathbf{T}$ 


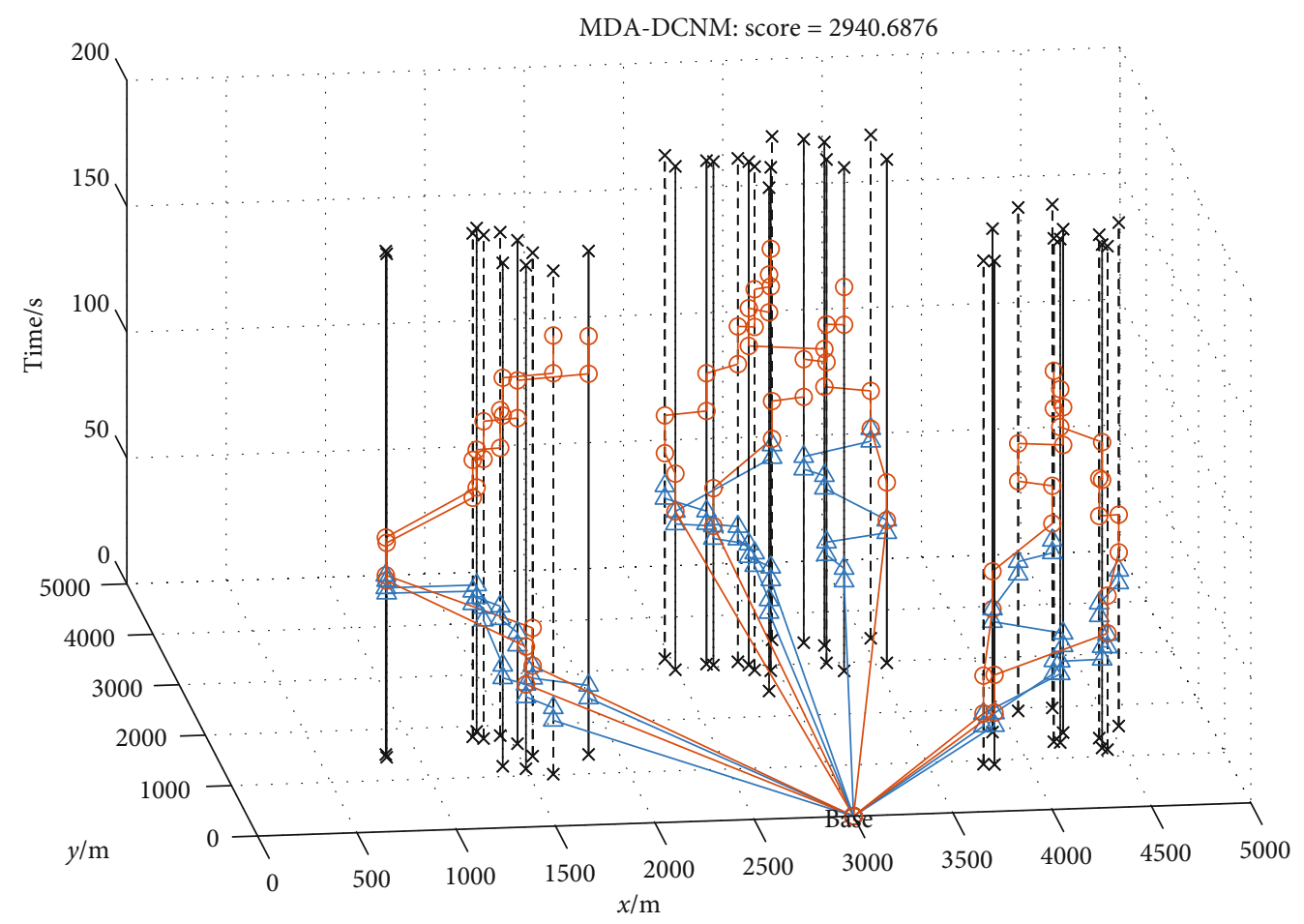

FIgURE 5: Task assignment results.

(2) The targets in $N_{C}=3$ subclusters are nonintersection. That is, $\mathbf{T}_{1} \cap \mathbf{T}_{2}=\varnothing, \mathbf{T}_{1} \cap \mathbf{T}_{3}=\varnothing$, and $\mathbf{T}_{2} \cap \mathbf{T}_{3}=\varnothing$

Thus, the targets in $N_{C}=3$ subclusters satisfy the nonintersection and complete demands of the hierarchical task assignment model in (24)-(26).

Then, the central control station applies negotiation mechanism to assign different UAV teams to $N_{C}=3$ subclusters.

Assume that the limited task capacities of R-UAVs are

$$
\mathbf{L}_{t}^{R}=\left\{L_{t}^{1}, L_{t}^{2}, \cdots, L_{t}^{7}\right\}=\{8,9,9,10,10,7,7\}
$$

The $N_{C}=3$ UAV teams can be formed based on Algorithm 2 .

$$
\begin{gathered}
\mathbf{U}_{1}=\left\{U_{1}^{S}, U_{2}^{S}, U_{4}^{R}, U_{7}^{R}\right\}, \\
\mathbf{U}_{2}=\left\{U_{3}^{S}, U_{4}^{S}, U_{3}^{R}, U_{1}^{R}\right\}, \\
\mathbf{U}_{3}=\left\{U_{5}^{S}, U_{6}^{S}, U_{7}^{S}, U_{5}^{R}, U_{2}^{R}, U_{6}^{R}\right\} .
\end{gathered}
$$

Similarly, the UAVs in $N_{C}=3$ subclusters satisfy the nonintersection and complete demands of the hierarchical task assignment model in (21)-(23).

Therefore, MDA-DCNM decomposes the $N_{U}=14, N_{T}$ $=40$ task assignment problem into $N_{C}=3$ nonintersection and complete small-scale task assignment problems.

The corresponding elements of $N_{C}=3$ subproblems are shown in Table 4 , where $T_{q}, U_{q}\left(q=1,2, \cdots, N_{C}\right)$ separately represents the target and UAV sets of $q_{\mathrm{th}}$ submodel, R $e_{q}^{R}$ represents the requirements for $R$ tasks, $L_{t}^{R}$ represents the limited capacity of R-UAVs, and $\gamma_{q}, C h_{q}$ separately represents the residual task capacities and cluster heads of $N_{C}=3$ submodels.

In Table 4, the large-scale task assignment model has been decomposed into $N_{C}=3$ submodels. E.g., in the $1^{\text {st }}$ submodel, UAVs $\left\{U_{1}^{S}, U_{2}^{S}, U_{4}^{R}, U_{7}^{R}\right\}$ are assigned to perform tasks $\left\{T_{1}, T_{2}, \cdots, T_{12}\right\}$. The requirement of $R$ tasks is $\left\|\mathbf{T}_{1}\right\|$ $=12$; the task capacities of R-UAVs $U_{4}^{R}, U_{7}^{R}$ are separately $\{10,7\}$. Apparently, the residual task capacities in the $1^{\text {st }}$ submodel are $\gamma_{1}=10+7-12=5$. Through the DS fusion method, $U_{2}^{S}$ is selected as the cluster head of the $1^{\text {st }}$ submodel.

Accordingly, $N_{C}=3$ submodels are established. Considering the time coupling constraints of tasks and the heterogeneity of UAVs in these subproblems, CBBA-TCC is adopted to produce effective and conflict-free task assignment solutions.

Figure 5 shows the task assignment results of MDADCNM. In Figure 5, $N_{U}=14$ UAVs from the same base need to perform S\&R mission on $N_{T}=40$ targets. The $x$-axis and $y$ -axis represent the locations of targets; the $z$-axis is the time. By contrast to Figure 4, the black " $x$ " represents the locations of targets, the black dotted lines indicate the time windows of targets: $[0,200]$ s. " $\Delta, \bigcirc$ " separately represent $S$ and $R$ tasks, and their corresponding $z$-axis values are their execution time. Blue and red segments separately represent the task performing process of $S$-UAVs and $R$-UAVs.

Figure 6 shows the UAV schedules of MDA-DCNM. In Figure 6 , the blue and red segments separately represent that 


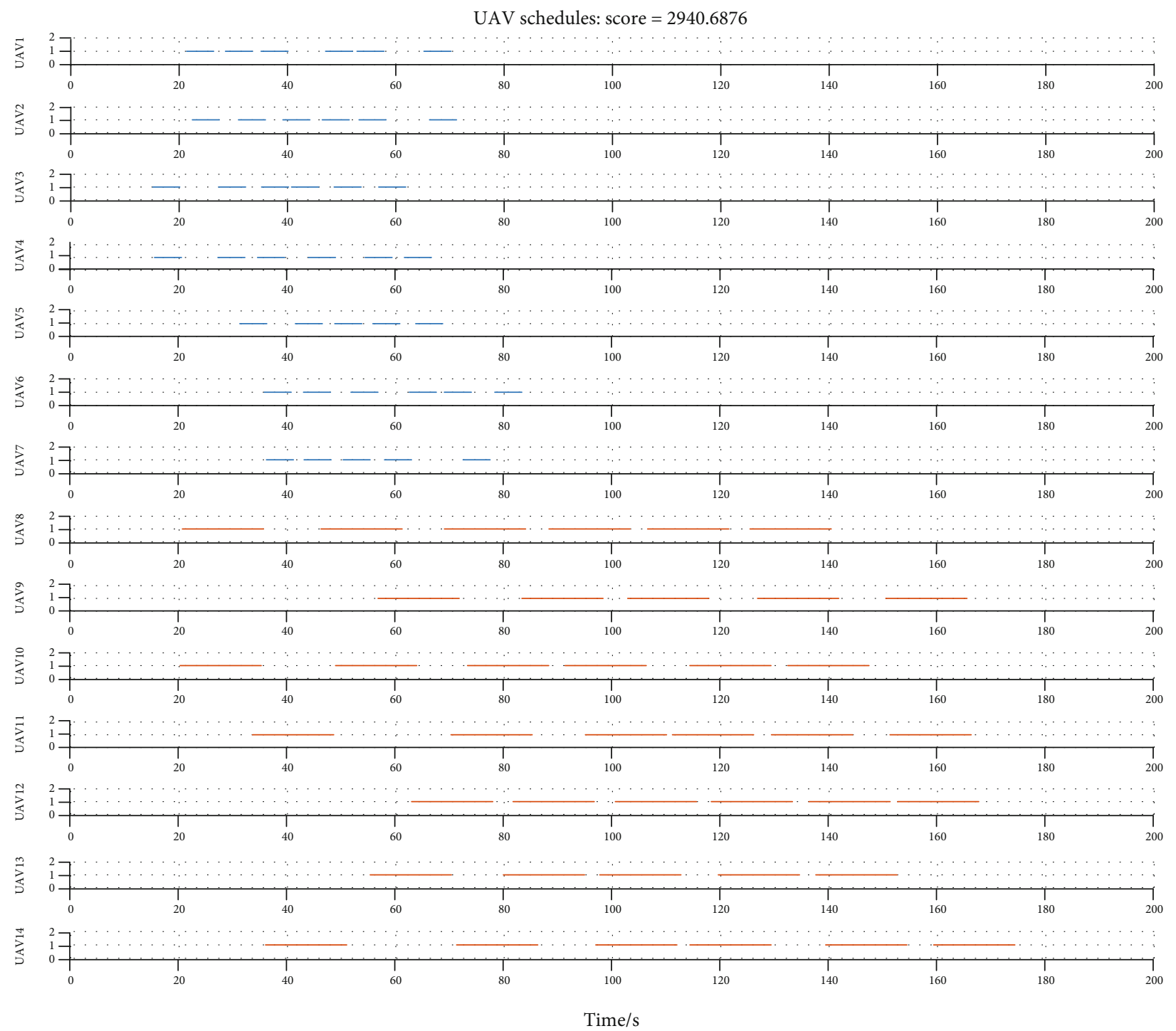

FIgURE 6: UAV schedules.

TABle 5: Scores of two algorithms under different target distributions.

\begin{tabular}{lcc}
\hline \multirow{2}{*}{ Target distributions } & \multicolumn{2}{c}{ Algorithms } \\
\hline$N_{C}=2$ & 2785.6575 & CBBA-TCC \\
$N_{C}=3$ & 3018.6522 & 2835.3650 \\
$N_{C}=4$ & 2012.4958 & 1952.2485 \\
$N_{C}=5$ & 2658.5116 & 2344.8481 \\
$N_{C}=6$ & 2504.3999 & 2456.2380 \\
\hline
\end{tabular}

the corresponding UAVs are performing $S$ and $R$ tasks, and the length of each segment indicates the execution duration of corresponding task.

We can see from Figures 5 and 6 that:

(1) Figure 5 reveals that only $S$ tasks $(\Delta)$ are assigned to $S$ -UAVs (blue), while only $R$ tasks $(\bigcirc)$ are assigned to
$R$-UAVs (red). That is, the constraint on different capabilities of UAVs is satisfied

(2) (72) shows that the limited task capacities of R-UAVs are $\mathbf{L}_{t}^{R}=\left\{L_{t}^{1}, L_{t}^{2}, \cdots, L_{t}^{7}\right\}=\{8,9,9,10,10,7,7\}$. Figure 6 shows that for each $R-\mathrm{UAV}$, the number of assigned tasks is less than its task capacity. Thus, the constraint on limited payloads of $R$-UAVs is satisfied

(3) Figure 5 reflects that both $S$ and $R$ tasks of each target are effectively assigned. That is, the task completion constraint in (13) is satisfied

(4) Figure 5 indicates that for each target, its $R$ task is always performed after the completion of $S$ task. Thus, the task precedence constraint in (14)-(16) is satisfied

(5) Figure 6 directly reflects that all tasks are performed within time window $[0,200] \mathrm{s}$. Thus, the timesensitive constraint in (14)-(16) is satisfied 
TABLE 6: Convergence performance of two algorithms under different target distributions.

\begin{tabular}{lcccr}
\hline \multirow{2}{*}{ Target distributions } & \multicolumn{2}{c}{ Algorithms } & \multicolumn{2}{c}{ CBBA-TCC } \\
& Convergence time & Communication amount & Convergence time & Communication amount \\
\hline$N_{C}=2$ & $1.1751 \mathrm{~s}$ & 35 & $4.7843 \mathrm{~s}$ & 40 \\
$N_{C}=3$ & $0.4230 \mathrm{~s}$ & 25 & $4.8277 \mathrm{~s}$ & 47 \\
$N_{C}=4$ & $0.1798 \mathrm{~s}$ & 23 & $2.7072 \mathrm{~s}$ & 36 \\
$N_{C}=5$ & $0.0627 \mathrm{~s}$ & 14 & $3.8543 \mathrm{~s}$ & 42 \\
$N_{C}=6$ & $0.0197 \mathrm{~s}$ & 9 & $4.4150 \mathrm{~s}$ & 40 \\
\hline
\end{tabular}

TABLE 7: Scores of two algorithms under different mission sizes.

\begin{tabular}{lcc}
\hline \multirow{2}{*}{ Size of mission area } & \multicolumn{2}{c}{ Algorithms } \\
\hline $5 \mathrm{~km} \times 5 \mathrm{~km}$ & 5529.9229 & 5245.2512 \\
$10 \mathrm{~km} \times 5 \mathrm{~km}$ & 4917.3263 & 4513.8468 \\
$10 \mathrm{~km} \times 10 \mathrm{~km}$ & 4834.5632 & 4413.3940 \\
$20 \mathrm{~km} \times 10 \mathrm{~km}$ & 4335.3501 & 3958.5015 \\
$20 \mathrm{~km} \times 20 \mathrm{~km}$ & 3311.3769 & 3189.3496 \\
\hline
\end{tabular}

Overall, the proposed MDA-DCNM can produce effective and conflict-free solutions in the large-scale S\&R scenarios with dispersed clustering targets.

Besides, the multi-UAV system relies on communications and negotiations to produce feasible and conflict-free task assignment solution in S\&R scenarios. We can derive from Figure 5 that MDA-DCNM successfully decomposes the large-scale task assignment problem into several smallscale submodels, where UAVs only communication with corresponding UAVs in the same subteam to realize the assignment of tasks in corresponding submodel. Therefore, compared with direct task assignment strategy, the proposed MDA-DCNM greatly reduces the communication amount and cruise path of UAVs.

4.2. Different Distributions of Targets. Suppose that $N_{U}=14$ , $N_{S}=7, N_{R}=7$, and $N_{T}=40$ task assignment problem exists in $5 \mathrm{~km} \times 5 \mathrm{~km}$ mission area, and the randomly generated targets are dispersed clustering at $N_{C}=2-6$ locations. Other simulation parameters are the same as Section 4.1. Based on 100 Monte Carlo simulations, the score values and convergence performance of MDA-DCNM and CBBA-TCC are respectively shown in Tables 5 and 6 .

We can see from Table 5 that under different target distribution situations, the proposed MDA-DCNM always produces solutions with higher score values than CBBA-TCC. Table 6 reveals that the proposed MDA-DCNM always has less convergence time and communication amount than CBBA-TCC. Therefore, compared with CBBA-TCC, the decomposition process of MDA-DCNM not only greatly reduces the cruise consumption of UAVs but also greatly reduces the required communication burden. That is, the proposed MDA-DCNM can produce feasible and conflictfree solution with higher scores and less convergence time.

Besides, we can see from Table 6 that for the same number of targets $N_{T}=40$, the more dispersed these targets are, the smaller these submodels are, and the less convergence time and communication amount the proposed MDADCNM needs. Thus, the decomposition process of the large-scale task assignment model can be strengthened by adjusting the parameters MinPts and $\varepsilon$ of density clustering algorithm to obtain finer dimension reduction results, which can help the multi-UAV system get the task assignment solutions more quickly.

4.3. Different Size of Mission Area. Suppose that the randomly generated $N_{T}=40$ targets are dispersed clustering at $N_{C}=3$ locations in different size of mission area, $N_{U}=14$, $N_{S}=7$, and $N_{R}=7$ UAVs need to perform S\&R mission on these targets. Based on 100 Monte Carlo simulations, the score values and convergence performance of MDADCNM and CBBA-TCC are respectively shown in Tables 7 and 8.

We can see from Tables 7 and 8 that under different size of mission area, the proposed MDA-DCNM always produces solutions with higher score, less convergence time, and less communication amount than CBBA-TCC.

Besides, Table 8 shows that the convergence time and communication amount of CBBA-TCC in $20 \mathrm{~km} \times 20 \mathrm{~km}$ mission area are separately $178 \mathrm{~s}$ and 522 times, which is not suitable for real-time task assignment requirement. The decomposition process of MDA-DCNM greatly reduces the required convergence time and communication amount to $0.8 \mathrm{~s}$ and 26 times, which effectively achieves the real-time requirement in $\mathrm{S} \& \mathrm{R}$ scenarios.

4.4. Different Model Scales. To further discuss the superiority of the proposed MDA-DCNM, 100 Monte Carlo simulations are conducted in different scales of mission models. The mission area is $10 \mathrm{~km} \times 10 \mathrm{~km}$, and targets are gathering at $N_{C}$ $=3$ locations. Tables 9 and 10 separately give the score values and convergence performance of MDA-DCNM and CBBATCC.

Apparently, Tables 9 and 10 show that under different S\&R mission scales, the proposed MDA-DCNM always produces solutions with higher score, less convergence time, and less communication amount than CBBA-TCC. 
TABLE 8: Convergence performance of two algorithms under different mission sizes.

\begin{tabular}{lcccc}
\hline \multirow{2}{*}{ Size of mission area } & \multicolumn{2}{c}{ Algorithms } & \multicolumn{2}{c}{ CBBA-TCC } \\
& Convergence time & Communication amount & Convergence time & Communication amount \\
\hline $5 \mathrm{~km} \times 5 \mathrm{~km}$ & $0.9864 \mathrm{~s}$ & 27 & $35.9210 \mathrm{~s}$ & 59 \\
$10 \mathrm{~km} \times 5 \mathrm{~km}$ & $0.7505 \mathrm{~s}$ & 25 & $40.3384 \mathrm{~s}$ & 57 \\
$10 \mathrm{~km} \times 10 \mathrm{~km}$ & $0.6596 \mathrm{~s}$ & 20 & $43.5121 \mathrm{~s}$ & 59 \\
$20 \mathrm{~km} \times 10 \mathrm{~km}$ & $0.4255 \mathrm{~s}$ & 21 & $28.6688 \mathrm{~s}$ & 60 \\
$20 \mathrm{~km} \times 20 \mathrm{~km}$ & $0.8207 \mathrm{~s}$ & 26 & $178.5785 \mathrm{~s}$ & 522 \\
\hline
\end{tabular}

TABle 9: Scores of two algorithms under different model scales.

\begin{tabular}{lcc}
\hline \multirow{2}{*}{ Model scales } & \multicolumn{2}{c}{ Algorithms } \\
\hline$N_{U}=10, N_{T}=40$ & 4339.1163 & CBBA-TCC \\
$N_{U}=20, N_{T}=40$ & 4611.0305 & 3861.5895 \\
$N_{U}=20, N_{T}=60$ & 6427.3621 & 6269.6223 \\
$N_{U}=20, N_{T}=80$ & 8782.4890 & 7379.5571 \\
$N_{U}=30, N_{T}=60$ & 6987.2419 & 6544.5443 \\
$N_{U}=30, N_{T}=80$ & 8986.3012 & 8189.2356 \\
$N_{U}=30, N_{T}=100$ & 10636.9559 & 9919.5550 \\
$N_{U}=40, N_{T}=80$ & 9286.5631 & 8527.9601 \\
$N_{U}=40, N_{T}=100$ & 11108.0804 & 10466.3619 \\
\hline
\end{tabular}

Besides, Table 10 reveals that when the whole task assignment model is clustered as $N_{C}=3$ sub-models, even the convergence time of MDA-DCNM cannot always meet the realtime task assignment requirement of S\&R mission. E.g., when $N_{U}=40, N_{T}=100$, the average numbers of UAVs and targets in $N_{C}=3$ submodels are separately 13 and 33 . Under this circumstance, these submodels cannot be solved quickly. Accordingly, we can derive that further refinement of model decomposition process can increase the number of submodels, reduce the scale of submodels, and thus shorten the convergence time of MDA-DCNM. This just echoes the analyses of Table 6: the larger the whole task assignment model is decomposed (larger $N_{C}$ ), the faster the proposed MDA-DCNM converges.

4.5. R-UAV Has Unbalanced Resources. The above simulations have proved the feasibility and superiority of MDADCNM. Under the circumstance of UAVs' limited and unbalanced resources, a few tasks cannot be effectively allocated through their corresponding UAV teams. Thus, ATSUT in hierarchical task assignment strategy is triggered to realize the mission coverage in the large-scale S\&R scenarios.

Suppose that $N_{T}=40$ targets randomly gather at $N_{C}=3$ locations in $5 \mathrm{~km} \times 5 \mathrm{~km}$ mission area, $N_{U}=14$ UAVs need to perform $\mathrm{S} \& \mathrm{R}$ mission on all targets within time window $[0,200]$ s. The limited and unbalanced task capacities of RUAVs are $\mathbf{L}_{t}^{R}=\left\{L_{t}^{1}, L_{t}^{2}, \cdots, L_{t}^{7}\right\}=\{4,7,8,6,7,6,4\}$, and other relevant simulation parameters are the same as Section 4.1.
Through the model decomposition process of MDA-DCNM, $N_{C}=3$ submodels are obtained. The corresponding elements of these submodels are shown in Table 11.

In Table 11, the large-scale task assignment model has been decomposed into $N_{C}=3$ submodels.

In the $1^{\text {st }}$ and $3^{\text {rd }}$ submodels, the residual task capacities are separately $\gamma_{1}=6+7-12=1$ and $\gamma_{3}=8+6+4-16=2$. Obviously, the small-scale task assignment problems in the $1^{\text {st }}$ and $3^{\text {rd }}$ models can be handled well.

In the $2^{\text {nd }}$ submodel, the residual task capacity is $\gamma_{2}=7$ $+4-12=-1$. That is, in the $2^{\text {nd }}$ submodel, the task capacity of UAV team is less than the requirement for $R$ tasks. Thus, due to limited and unbalanced task capacities of R-UAVs, the UAV team in the $2^{\text {nd }}$ submodel cannot guarantee the effective assignment of all tasks in the subcluster.

Under this circumstance, the task assignment results and UAV schedules of MDA-DCNM are respectively shown in Figures 7 and 8.

Similarly to Figures 5 and 6, we can derive from Figures 7 and 8 that the task assignment solutions of MDA-DCNM satisfy the constraint on different capabilities of UAVs, constraint on limited payloads of R-UAVs, the task completion constraint, task precedence constraint, and time-sensitive constraint. Hence, MDA-DCNM can produce feasible and conflict-free task assignment solutions in the large-scale S\&R scenarios.

Table 11 reflects that the residual task capacity of the $2^{\text {nd }}$ submodel is $\gamma_{2}=-1$. Obviously, due to unbalanced task capacities of R-UAVs, the R-UAVs in the $2^{\text {nd }}$ submodel do not have sufficient resources to perform all corresponding $R$ tasks.

Then, in Figure 7, the purple "*” represents the unallocated target $T_{14}$; the purple segment indicates its time window. Figure 7 reveals that due to the limited task capacities of R-UAVs in the $2^{\text {nd }}$ submodel, the target $T_{14}$ in the $2^{\text {nd }}$ submodel is not assigned.

After the central control station receives the feedback information from cluster heads $U_{2}^{S}, U_{3}^{S}, U_{6}^{S}$ of $N_{C}=3$ submodels, ATS-UT is triggered to assign the unallocated target $T_{14}$ and further achieve the mission coverage of S\&R scenarios.

After receiving the task announcement from the central control station, the $1^{\text {st }}$ and $3^{\text {rd }}$ UAV teams will apply CBBAPR to assign the unallocated target $T_{14}$. Based on CBBA-PR, the score increments will be sent to the central control station as the biddings for the unallocated target $T_{14}$. 
TABLE 10: Convergence performance of two algorithms under different model scales.

\begin{tabular}{lcccc}
\hline \multirow{2}{*}{ Model scales } & \multicolumn{2}{c}{ Algorithms } & \multicolumn{2}{c}{ CBBA-TCC } \\
& Convergence time & Communication amount & Convergence time & Communication amount \\
\hline$N_{U}=10, N_{T}=40$ & $0.3526 \mathrm{~s}$ & 20 & $16.5170 \mathrm{~s}$ & 41 \\
$N_{U}=20, N_{T}=40$ & $0.9182 \mathrm{~s}$ & 29 & $39.3825 \mathrm{~s}$ & 58 \\
$N_{U}=20, N_{T}=60$ & $3.3564 \mathrm{~s}$ & 36 & $105.5134 \mathrm{~s}$ & 70 \\
$N_{U}=20, N_{T}=80$ & $8.5320 \mathrm{~s}$ & 42 & $256.0116 \mathrm{~s}$ & 83 \\
$N_{U}=30, N_{T}=60$ & $5.2269 \mathrm{~s}$ & 41 & $201.1032 \mathrm{~s}$ & 81 \\
$N_{U}=30, N_{T}=80$ & $14.3678 \mathrm{~s}$ & 45 & $303.2863 \mathrm{~s}$ & 90 \\
$N_{U}=30, N_{T}=100$ & $25.5909 \mathrm{~s}$ & 51 & $590.8205 \mathrm{~s}$ & 105 \\
$N_{U}=40, N_{T}=80$ & $22.8850 \mathrm{~s}$ & 54 & $467.8749 \mathrm{~s}$ & 117 \\
$N_{U}=40, N_{T}=100$ & $36.1597 \mathrm{~s}$ & 58 & $858.5061 \mathrm{~s}$ & 121 \\
\hline
\end{tabular}

TABle 11: $N_{C}=3$ submodels with unbalanced capacities of $R$-UAVs.

\begin{tabular}{lccc}
\hline Elements & $1^{\text {st }}$ subproblem & $2^{\text {nd }}$ subproblem & $3^{\text {rd }}$ subproblem \\
\hline $\mathbf{T}_{q}$ & $\left\{T_{1}, T_{2}, \cdots, T_{12}\right\}$ & $\left\{T_{13}, T_{14}, \cdots, T_{24}\right\}$ & $\left\{T_{25}, T_{26}, \cdots, T_{40}\right\}$ \\
$R e_{q}^{R}$ & 12 & 12 & 16 \\
$\mathbf{U}_{q}$ & $\left\{U_{1}^{S}, U_{2}^{S}, U_{4}^{R}, U_{5}^{R}\right\}$ & $\left\{U_{3}^{S}, U_{4}^{S}, U_{2}^{R}, U_{7}^{R}\right\}$ & $\left\{U_{5}^{S}, U_{6}^{S}, U_{7}^{S}, U_{3}^{R}, U_{6}^{R}, U_{1}^{R}\right\}$ \\
$\mathbf{L}_{t}^{R}$ & $\{6,7\}$ & $\{7,4\}$ & $\{8,6,4\}$ \\
$\gamma_{q}$ & 1 & -1 & 2 \\
$C h_{q}$ & $U_{2}^{S}$ & $U_{3}^{S}$ & $U_{6}^{S}$ \\
\hline
\end{tabular}

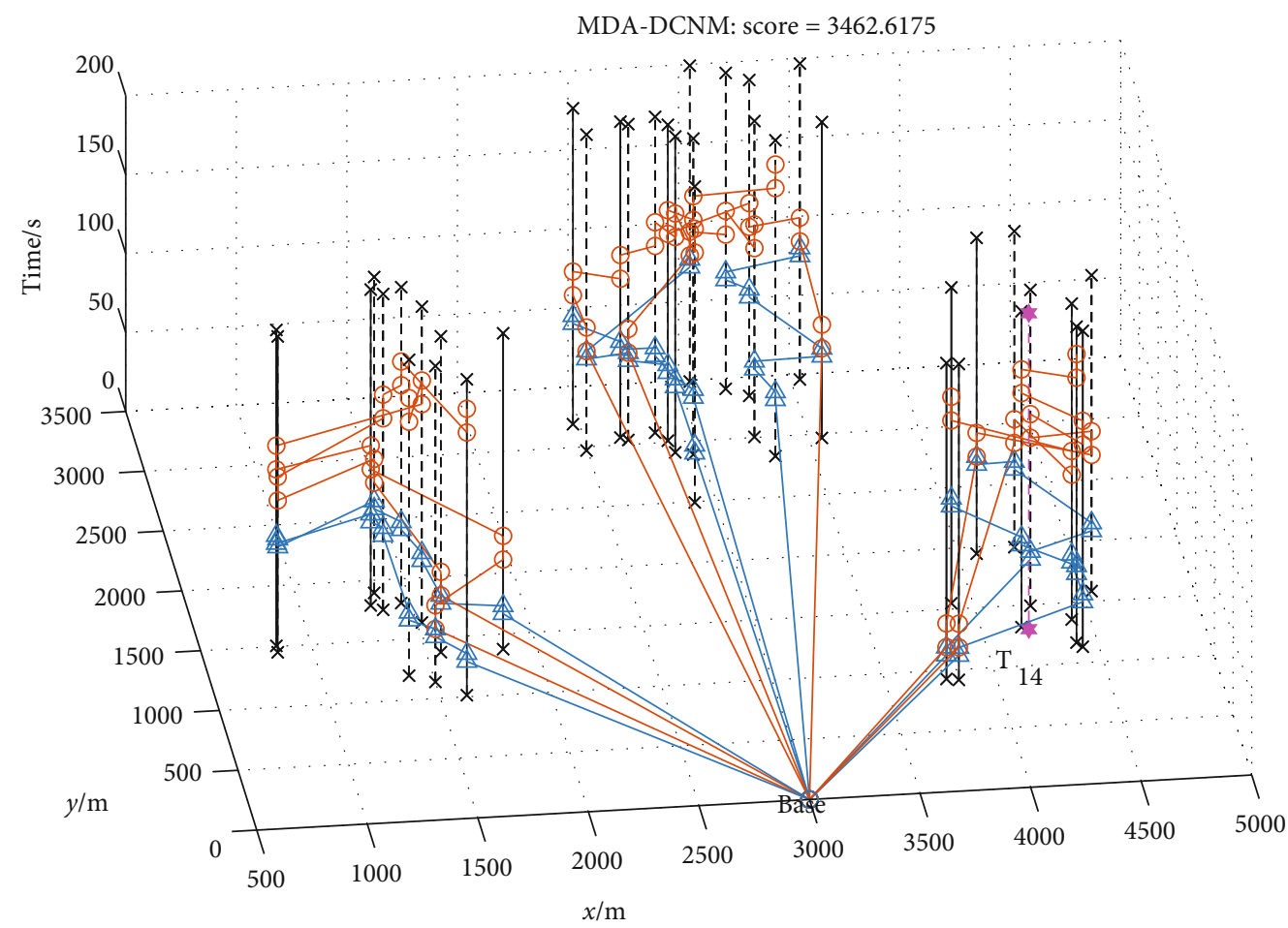

FIgURE 7: Task assignment results of MDA-DCNM with unbalanced capacities of $R$-UAVs. 


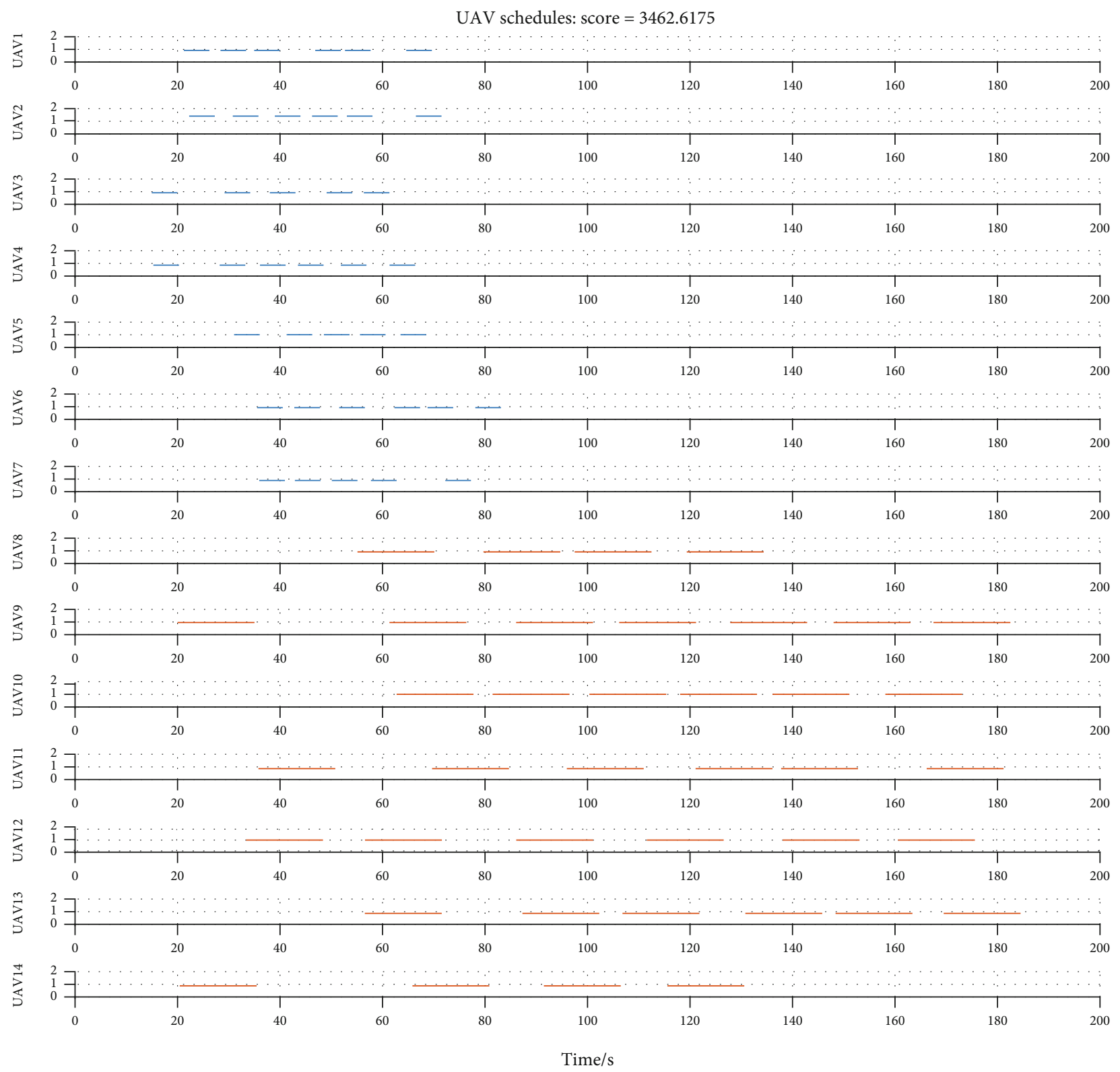

FIgURE 8: UAV schedules of MDA-DCNM with unbalanced capacities of $R$-UAVs.

The auction information of the $1^{\text {st }}$ and $3^{\text {rd }}$ UAV teams are respectively shown in Figures 9 and 10.

Figure 9 only gives the task performing information of the $1^{\text {st }}$ UAV team. On the basis of its original task assignment solution in Figure 7 , the $1^{\text {st }}$ UAV team will apply CBBA-PR to realize the effective assignment of the unallocated target $T_{14}$. Figure 9 shows that for the $1^{\text {st }}$ UAV team, the score increment of $T_{14}$ is 140.5928 . Then, the cluster head of the $1^{\text {st }}$ UAV team $U_{2}^{S}$ will send the score increment 140.5928 to the central control station as its auction value for $T_{14}$.

Figure 10 only gives the task performing information of the $3^{\text {rd }}$ UAV team. Similarly, Figure 10 reveals that for the $3^{\text {rd }} \mathrm{UAV}$ team, the score increment of $T_{14}$ is 261.9860 . Then, the cluster head of the $3^{\text {rd }} \mathrm{UAV}$ team $U_{3}^{S}$ will send the score increment 261.9860 to the central control station as its auction value for $T_{14}$.

Finally, as the objective of the task assignment model is to maximize the global reward when satisfying multiple constraints, the central control station will chose the $3^{\text {rd }} \mathrm{UAV}$ team with higher bidding to perform the $S$ and $R$ tasks of the unallocated target $T_{14}$.

Accordingly, the unallocated target $T_{14}$ has been well assigned through ATS-UT. Hence, ATS-UT successfully guarantees the mission coverage in the large-scale S\&R scenarios even UAVs has limited and unbalanced sources.

Overall, MDA-DCNM can realize the feasible decomposition of the large-scale task assignment model, which availably reduces the required communication amount and 


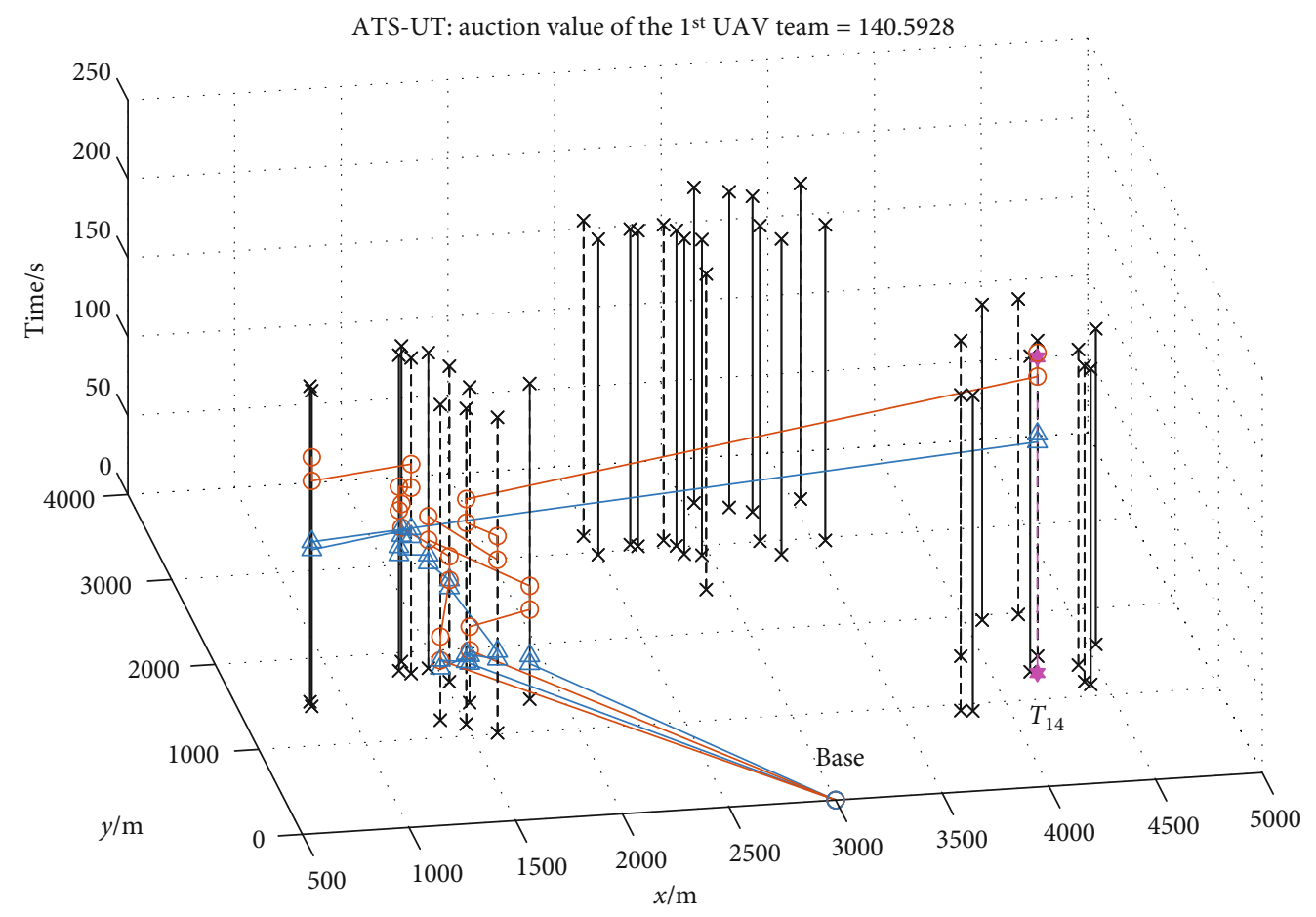

Figure 9: CBBA-PR auction information of the $1^{\text {st }}$ UAV team.

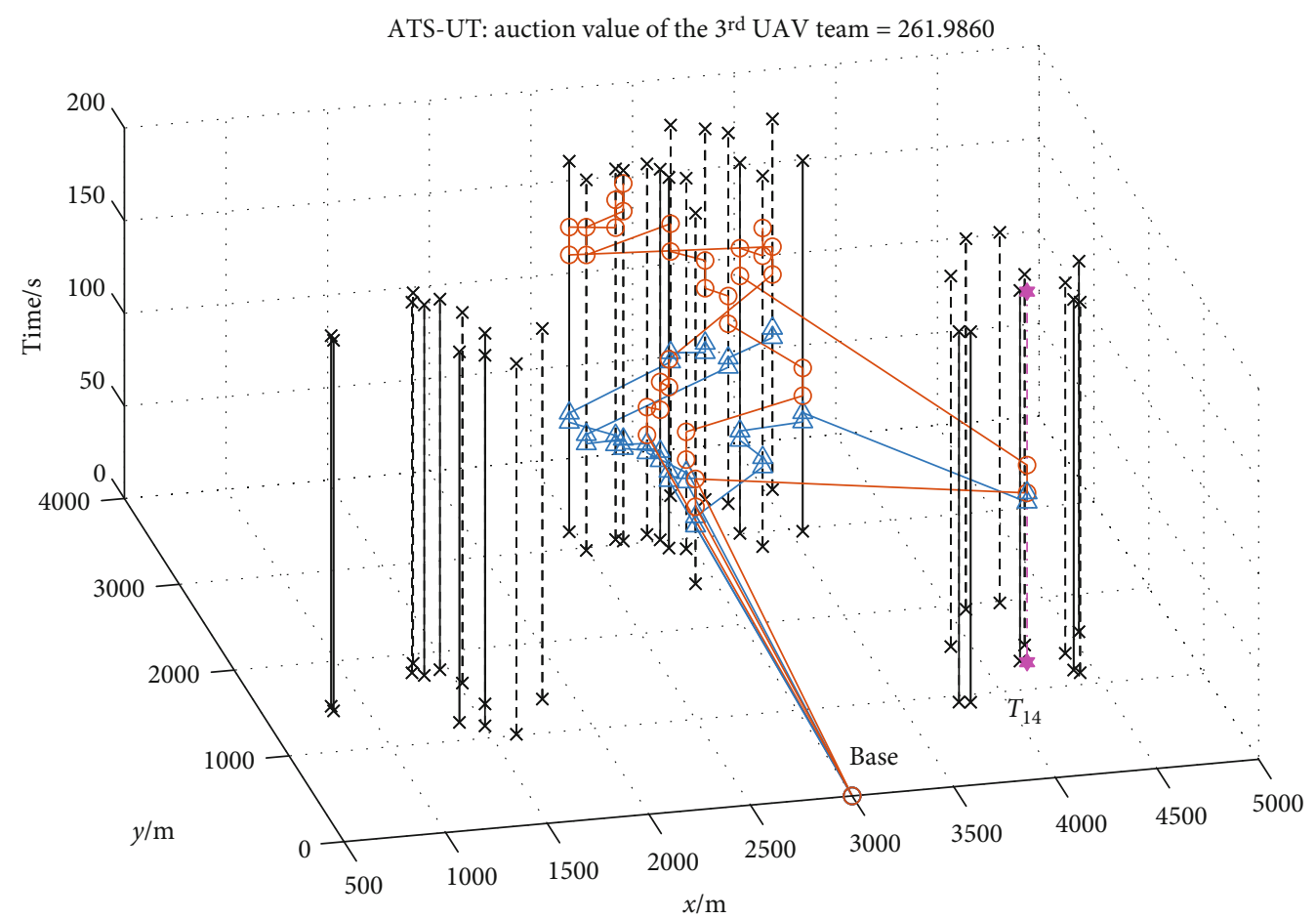

FIGURE 10: CBBA-PR auction information of the $3^{\text {rd }}$ UAV team.

computational burden of the multi-UAV system. When UAVs have limited and unbalanced sources, a few task cannot be effectively assigned. Then, ATS-UT is triggered to guarantee the efficient assignment of unallocated tasks. Therefore, the effectiveness of the proposed hierarchical task assignment strategy has been well illustrated. 


\section{Conclusions}

To solve the multi-UAV multitask assignment problem in the large-scale S\&R scenarios with dispersed clustering targets, this paper proposes a hierarchical task assignment strategy. Firstly, the model decoupling algorithm based on density clustering and negotiation mechanism is proposed to decompose the large-scale task assignment model with dispersed clustering targets into several nonintersection and complete small-scale task assignment models. The model decomposition process greatly reduces the required computational amount and communication cost. Then, a cluster head selection based on DS fusion decision is raised to select rational cluster head for each UAV team according to the overall analyses of UAVs' four attributes including the mission execution time, the number of undertaken tasks, the total score values, and the residual task capacities. At last, considering the unallocated targets caused by UAVs' limited and unbalanced resources, an auction-based task-sharing scheme among UAV teams is raised to achieve the effective assignment of unallocated tasks and further guarantee the mission coverage in the large-scale S\&R scenarios. Simulation results comprehensively prove that under the large-scale task assignment model with dispersed clustering targets, the proposed hierarchical task assignment strategy can quickly generate effective, reliable, and conflict-free task assignment solutions that conform to multiple constraints.

In the future research, we will study the application of the proposed hierarchical task assignment strategy in the urban S\&R scenarios. There are many buildings, trees, and other obstacles in urban environment. Thus, the next study will focus on how to ensure the effectiveness of the hierarchical task assignment strategy under complex urban environment.

\section{Data Availability}

The data, models, and code used during the study are available from the corresponding author by request.

\section{Conflicts of Interest}

The authors declare that there is no conflict of interests regarding the publication of this paper.

\section{Acknowledgments}

The paper is funded by the National Natural Science Foundation of China (No. 61701134, No. 51809056) and the Fundamental Research Funds for the Central Universities (No. 3072020CF0811).

\section{References}

[1] M. Zhu, X. du, X. Zhang, H. Luo, and G. Wang, "Multi-UAV rapid-assessment task-assignment problem in a postearthquake scenario," IEEE Access, vol. 7, pp. 74542-74557, 2019.

[2] K. Harikumar, J. Senthilnath, and S. Sundaram, "Multi-UAV oxyrrhis marina-inspired search and dynamic formation con- trol for forest firefighting," IEEE Transactions on Automation Science and Engineering, vol. 16, no. 2, pp. 863-873, 2018.

[3] H. X. Pham, H. M. La, D. Feil-Seifer, and M. C. Deans, "A distributed control framework of multiple unmanned aerial vehicles for dynamic wildfire tracking," IEEE Transactions on Systems, Man, and Cybernetics: Systems, vol. 50, no. 4, pp. 1537-1548, 2018.

[4] H. Wu, H. Li, R. Xiao, and J. Liu, "Modeling and simulation of dynamic ant colony's labor division for task allocation of UAV swarm," Physica A: Statistical Mechanics and its Applications, vol. 491, pp. 127-141, 2018.

[5] Y. Zhang, W. Feng, G. Shi, F. Jiang, M. Chowdhury, and S. H. Ling, "UAV swarm mission planning in dynamic environment using consensus-based bundle algorithm," Sensors, vol. 20, no. 8, p. 2307, 2020.

[6] W. Yao, N. Qi, N. Wan, and Y. Liu, "An iterative strategy for task assignment and path planning of distributed multiple unmanned aerial vehicles," Aerospace Science and Technology, vol. 86, pp. 455-464, 2019.

[7] Y. Chen, D. Yang, and J. Yu, "Multi-UAV task assignment with parameter and time-sensitive uncertainties using modified two-part wolf pack search algorithm," IEEE Transactions on Aerospace and Electronic Systems, vol. 54, no. 6, pp. 28532872, 2018.

[8] J. Wang, J. Wang, and H. Che, "Task assignment for multivehicle systems based on collaborative neurodynamic optimization," IEEE Transactions on Neural Networks and Learning Systems, vol. 31, no. 4, pp. 1145-1154, 2020.

[9] S. J. Rasmussen and T. Shima, "Tree search algorithm for assigning cooperating UAVs to multiple tasks," International Journal of Robust and Nonlinear Control, vol. 18, no. 2, pp. 135-153, 2008.

[10] D. K. Ahner and C. R. Parson, "Optimal multi-stage allocation of weapons to targets using adaptive dynamic programming," Optimization Letters, vol. 9, no. 8, pp. 1689-1701, 2015.

[11] Z. Zhen, Y. Chen, L. Wen, and B. Han, "An intelligent cooperative mission planning scheme of UAV swarm in uncertain dynamic environment," Aerospace Science and Technology, vol. 100, p. 105826, 2020.

[12] Z. Wang, M. Zheng, J. Guo, and H. Huang, "Uncertain UAV ISR mission planning problem with multiple correlated objectives," Journal of Intelligent \& Fuzzy Systems, vol. 32, no. 1, pp. 321-335, 2017.

[13] Y. Khosiawan, Y. Park, I. Moon, J. M. Nilakantan, and I. Nielsen, "Task scheduling system for UAV operations in indoor environment," Neural Computing and Applications, vol. 31, no. 9, pp. 5431-5459, 2019.

[14] Z. Zhu, B. Tang, and J. Yuan, "Multirobot task allocation based on an improved particle swarm optimization approach," International Journal of Advanced robotic systems, vol. 14, no. 3, 2017.

[15] Z. Wang, L. Liu, T. Long, and Y. Wen, "Multi-UAV reconnaissance task allocation for heterogeneous targets using an opposition-based genetic algorithm with doublechromosome encoding," Chinese Journal of Aeronautics, vol. 31, no. 2, pp. 339-350, 2018.

[16] Z. Jia, J. Yu, X. Ai, X. Xu, and D. Yang, "Cooperative multiple task assignment problem with stochastic velocities and time windows for heterogeneous unmanned aerial vehicles using a genetic algorithm," Aerospace Science and Technology, vol. 76, pp. 112-125, 2018. 
[17] G. Xu, T. Long, Z. Wang, and L. Liu, "Target-bundled genetic algorithm for multi-unmanned aerial vehicle cooperative task assignment considering precedence constraints," Proceedings of the Institution of Mechanical Engineers, Part G: Journal of Aerospace Engineering, vol. 234, no. 3, pp. 760-773, 2020.

[18] X. Chen, P. Zhang, G. Du, and F. Li, “A distributed method for dynamic multi-robot task allocation problems with critical time constraints," Robotics and Autonomous Systems, vol. 118, pp. 31-46, 2019.

[19] H. Kurdi, M. F. AlDaood, S. al-Megren, E. Aloboud, A. S. Aldawood, and K. Youcef-Toumi, "Adaptive task allocation for multi-UAV systems based on bacteria foraging behaviour," Applied Soft Computing, vol. 83, p. 105643, 2019.

[20] T. Shima, S. J. Rasmussen, A. G. Sparks, and K. M. Passino, "Multiple task assignments for cooperating uninhabited aerial vehicles using genetic algorithms," Computers \& Operations Research, vol. 33, no. 11, pp. 3252-3269, 2006.

[21] M. Otte, M. J. Kuhlman, and D. Sofge, "Auctions for multirobot task allocation in communication limited environments," Autonomous Robots, vol. 44, no. 3-4, pp. 547-584, 2020.

[22] G. Oh, Y. Kim, J. Ahn, and H. L. Choi, "Market-based task assignment for cooperative timing missions in dynamic environments," Journal of Intelligent \& Robotic Systems, vol. 87, no. 1, pp. 97-123, 2017.

[23] W. Meng, Z. He, R. Su, P. K. Yadav, R. Teo, and L. Xie, “Decentralized multi-UAV flight autonomy for moving convoys search and track," IEEE Transactions on Control Systems Technology, vol. 25, no. 4, pp. 1480-1487, 2017.

[24] Y. Xiao and D. Zhang, "The command decision method of multiple UUV cooperative task assignment based on contract net protocol," Journal of Systems Science and Information, vol. 4, no. 4, pp. 379-390, 2016.

[25] H. L. Choi, L. Brunet, and J. P. How, "Consensus-based decentralized auctions for robust task allocation," IEEE transactions on robotics, vol. 25, no. 4, pp. 912-926, 2009.

[26] A. Whitbrook, Q. Meng, and P. W. H. Chung, "Reliable, distributed scheduling and rescheduling for time-critical, multiagent systems," IEEE Transactions on Automation Science and Engineering, vol. 15, no. 2, pp. 732-747, 2017.

[27] E. Nunes, M. Manner, H. Mitiche, and M. Gini, "A taxonomy for task allocation problems with temporal and ordering constraints," Robotics and Autonomous Systems, vol. 90, pp. 5570, 2017.

[28] K. S. Kim, H. Y. Kim, and H. L. Choi, "A bid-based grouping method for communication-efficient decentralized multiUAV task allocation," International Journal of Aeronautical and Space Sciences, vol. 21, no. 1, pp. 290-302, 2020.

[29] G. Binetti, D. Naso, and B. Turchiano, "Decentralized task allocation for surveillance systems with critical tasks," Robotics and Autonomous Systems, vol. 61, no. 12, pp. 1653-1664, 2013.

[30] J. Godoy and M. Gini, "Task Allocation for Spatially and Temporally Distributed Tasks," in Intelligent Autonomous Systems 12, pp. 603-612, Springer, Berlin, Heidelberg, 2013.

[31] J. C. Amorim, V. Alves, and E. P. de Freitas, "Assessing a swarm-GAP based solution for the task allocation problem in dynamic scenarios," Expert Systems with Applications, vol. 152, p. 113437, 2020.

[32] N. Geng, Q. Meng, D. Gong, and P. W. H. Chung, "How good are distributed allocation algorithms for solving urban search land rescue problems? A comparative study with centralized algorithms," IEEE Transactions on Automation Science and Engineering, vol. 16, no. 1, pp. 478-485, 2018.

[33] Y. Altshuler, A. Pentland, and A. M. Bruckstein, "Optimal Dynamic Coverage Infrastructure for Large-Scale Fleets of Reconnaissance UAVs," in Swarms and Network Intelligence in Search, pp. 207-238, Springer, Cham, 2018.

[34] X. Fu, P. Feng, B. Li, and X. Gao, "A two-layer task assignment algorithm for UAV swarm based on feature weight clustering," International Journal of Aerospace Engineering, vol. 2019, Article ID 3504248, 12 pages, 2019.

[35] H. Kumar, N. K. Chauhan, and P. K. Yadav, "A high performance model for task allocation in distributed computing system using k-means clustering technique," in Research Anthology on Architectures, Frameworks, and Integration Strategies for Distributed and Cloud Computing, pp. 12441268, IGI Global, 2021.

[36] F. Ye, J. Chen, Q. Sun, Y. Tian, and T. Jiang, “Decentralized task allocation for heterogeneous multi-UAV system with task coupling constraints," The Journal of Supercomputing, vol. 77, no. 1, pp. 111-132, 2021.

[37] S. Waharte and N. Trigoni, "Supporting search and rescue operations with UAVs," in 2010 International Conference on Emerging Security Technologies, pp. 142-147, Canterbury, UK, 2010 .

[38] A. A. R. Alsaeedy and E. K. P. Chong, " 5 G and UAVs for mission-critical communications: swift network recovery for search-and-rescue operations," Mobile Networks and Applications, vol. 25, no. 5, pp. 2063-2081, 2020.

[39] X. Duan, H. Liu, H. Tang, Q. Cai, F. Zhang, and X. Han, “A novel hybrid auction algorithm for multi-UAVs dynamic task assignment," IEEE Access, vol. 8, pp. 86207-86222, 2019.

[40] S. J. Nanda and G. Panda, "Design of computationally efficient density-based clustering algorithms," Data \& Knowledge Engineering, vol. 95, pp. 23-38, 2015.

[41] Y. Lv, T. Ma, M. Tang et al., "An efficient and scalable densitybased clustering algorithm for datasets with complex structures," Neurocomputing, vol. 171, pp. 9-22, 2016.

[42] H. Wang, D. Lin, J. Qiu, L. Ao, Z. du, and B. He, "Research on multiobjective group decision-making in condition-based maintenance for transmission and transformation equipment based on D-S evidence theory," IEEE Transactions on Smart Grid, vol. 6, no. 2, pp. 1035-1045, 2015.

[43] N. Nesa and I. Banerjee, "IoT-based sensor data fusion for occupancy sensing using Dempster-Shafer evidence theory for smart buildings," IEEE Internet of Things Journal, vol. 4, no. 5, pp. 1563-1570, 2017.

[44] M. E. Argyle, R. W. Beard, and D. W. Casbeer, Multi-Team Consensus Bundle Algorithm, vol. 24, no. 12, 2015, Springer, Netherlands, 2015.

[45] N. Buckman, H. L. Choi, and J. P. How, Partial Replanning for Decentralized Dynamic Task Allocation, no. article 0915, 2019AIAA Scitech 2019 Forum, 2019.

[46] J. Chen, X. Qing, F. Ye, K. Xiao, K. You, and Q. Sun, “Consensus-based bundle algorithm with local replanning for heterogeneous multi-UAV system in the time-sensitive and dynamic environment," The Journal of Supercomputing, 2021. 